\title{
Noncommutative Fourier transform for the Lorentz group via the Duflo map
}

\author{
Daniele Oriti \\ Max Planck Institute for Gravitational Physics (Albert Einstein Institute), \\ Am Mühlenberg 1, 14476 Potsdam-Golm, Germany \\ and Arnold-Sommerfeld-Center for Theoretical Physics, Ludwig-Maximilians-Universität, \\ Theresienstrasse 37, D-80333 München, Germany, EU \\ Giacomo Rosati \\ INFN, Sezione di Cagliari, Cittadella Universitaria, 09042 Monserrato, Italy
}

(Received 11 February 2019; published 13 May 2019)

\begin{abstract}
We defined a noncommutative algebra representation for quantum systems whose phase space is the cotangent bundle of the Lorentz group, and the noncommutative Fourier transform ensuring the unitary equivalence with the standard group representation. Our construction is from first principles in the sense that all structures are derived from the choice of quantization map for the classical system, the Duflo quantization map.
\end{abstract}

DOI: 10.1103/PhysRevD.99.106005

\section{INTRODUCTION}

Physical systems whose configuration or phase space is endowed with a curved geometry include, for example, point particles on curved spacetimes and rotor models in condensed matter theory, and they present specific mathematical challenges in addition to their physical interest. In particular, many important results have been obtained in the case in which their domain spaces can be identified with (Abelian) group manifolds or their associated homogeneous spaces.

Focusing on the case in which the nontrivial geometry is that of configuration space, identified with a Lie group, this is then reflected in the noncommutativity of the conjugate momentum space, whose basic variables correspond to the Lie derivatives acting on the configuration manifold, and that can be thus identified with the Lie algebra of the same Lie group. At the quantum level, this noncommutativity enters heavily in the treatment of the system. For instance, it prevents a standard $L^{2}$ representation of the Hilbert state space of the system in momentum picture, and may lead us to question whether a representation in terms of the (noncommutative) momentum variables exists at all. Further, if such a representation could be defined, one would also need a generalized notion

Published by the American Physical Society under the terms of the Creative Commons Attribution 4.0 International license. Further distribution of this work must maintain attribution to the author(s) and the published article's title, journal citation, and DOI. Funded by SCOAP . of (noncommutative) Fourier transform to establish the (unitary) equivalence between this new representation and the one based on $L^{2}$ functions on the group configuration space.

The standard way of dealing with this issue is indeed to renounce to a representation that makes direct use of the noncommutative Lie algebra variables, and to use instead a representation in terms of irreducible representations of the Lie group, i.e., to resort to spectral decomposition as the proper analogue of the traditional Fourier transform in flat space. In other words, instead of using some necessarily generalized eigenbasis of the noncommutative momentum (Lie algebra) variables, one uses a basis of eigenstates of a maximal set of commuting operators that are functions of the same. This strategy has of course many advantages, but it prevents the more geometrically transparent picture, more closely connected to the underlying classical system, that dealing directly with the noncommuting Lie algebra variables would provide.

In recent times, most work on these issues has been motivated by quantum gravity, where they turned out to play an important role. This happened in two domains. The first is effective (field theory) models of quantum gravity based (or inspired) by noncommutative geometry [1]. Such models have attracted a considerable interest because they offer a framework for much quantum gravity phenomenology [2]. From the physical point of view, these models are based on the hypothesis that some form of spacetime noncommutativity [1] constitutes a key remnant, at a macroscopic, continuum level, of more fundamental 
quantum gravity structures, and that one key way in which this manifests itself is by relaxing [3] (or making relative $[4,5])$ the usual notion of locality on which effective field theory (and most spacetime physics) is based. At the mathematical level, spacetime noncommutativity in these models is the conjugate manifestation of curvature in momentum space (and thus in phase space) [6]. In most of them, moreover, this curved momentum space is modeled by a Abelian Lie group (the phase space is therefore its cotangent bundle) or homogeneous spaces constructed from it, hence the relevance of the noncommutative Lie algebra representation and of the noncommutative Fourier transform for Lie groups (see e.g., [7-9]). The second domain is that of nonperturbative quantum gravity proper, more specifically the broad area comprising group field theory [10], spin foam models [11] and loop quantum gravity [12]. Here again the cotangent bundle of a Lie group plays a prominent role: it is the classical phase space of the variables associated to the (edges of the) lattices, which replace continuum spacetime manifolds and represent the basic interaction processes of the fundamental degrees of freedom of all these formalisms. The interpretation of these algebraic variables have, in turn, a discrete geometric interpretation, as discrete gravity connection (the group variables) and conjugate discrete metric variables (the Lie algebra elements). Once again, the Lie algebra representation and the noncommutative Fourier transform allow a representation of the formalisms in terms of more geometrically transparent data [13,14]; this, in turn, facilitates model building as well as the extraction of effective macroscopic physics.

In this article we are not going to deal with these physical aspects, although we will discuss them briefly. We focus instead on the formal aspects of the noncommutative Fourier transform. Among the many issues motivated by its intrinsic mathematical interest, it is important to clarify its general properties for arbitrary Lie groups and its explicit construction in the challenging case of noncompact ones. The mathematical foundations of the Lie algebra representation and of the noncommutative Fourier transform have been explored in very general terms, e.g., in [15]. A key result of that work has been to show how both the star product and the noncommutative plane waves that characterize fully such algebra representation and its equivalence with the other representations of the underlying quantum system, are uniquely determined by the initial choice of quantization map that defines the same quantum system starting from its classical phase space and algebra of observables. Moreover, this has been shown for arbitrary semisimple locally compact Lie groups. Thus, while the explicit constructions reported in the same paper only dealt with the compact group $\mathrm{SU}(2)$, the same procedure can be straightforwardly extended to the noncompact case. This is what we do in this paper, focusing on the Lorentz group $\operatorname{SL}(2, \mathbb{C})$ because of its general physical interest and because it plays in particular a key role in quantum gravity, in the context of spin foam models, group field theories as well as effective noncommutative spacetime models. The quantization map we use, which is the key technical input of the whole construction, is the Duflo map [16]. This has already found some applications in quantum gravity [17-22], and it had been used in [15] for the compact $\mathrm{SU}(2)$ case. For our present purposes, the mathematical features of this quantization map that makes it a preferred choice are its generality (it also can be applied to any semisimple Lie algebra) and its characterizing property of mapping the subalgebra of invariant functions (under the group action) on the classical phase space faithfully to a subalgebra of invariant operators in the quantum theory. This is mathematically desirable, but also physically crucial, in our opinion.

The plan of the paper is as follows. In the next section, we summarize the results of [15], for general Lie groups, which form the basis for our subsequent analysis and construction for the Lorentz group. In Sec. III we use the Duflo map to construct the Lie algebra representation of a quantum system whose classical phase space is the cotangent bundle of the Lorentz group, and then, in Sec. IV, using the same quantization map, we obtain the noncommutative Fourier transform that ensure its equivalence with the more traditional group representation, constructing explicitly the corresponding noncommutative plane waves. These are the key new results of this paper. We also offer a quick comparison with related results in the literature, concerning the definition and application of noncommutative methods for similar systems, both at the mathematical level and in quantum gravity contexts. Moreover, we show how our results generalize to the case of homogeneous spaces of the Lorentz group. Finally, in the last section, we show the application of our tools to the computation of the propagator of a single nonrelativistic point particle on the hyperboloid, as an example.

\section{BACKGROUND: LIE ALGEBRA REPRESENTATION AND NONCOMMUTATIVE FOURIER TRANSFORM}

The mathematical foundations of the noncommutative Fourier transform have been clarified in [15], and we base our present work on that analysis. We summarize its results here.

\section{A. Classical phase space and its quantization}

We consider physical systems whose configuration space is a Lie group $G$, and whose momentum space coincides with the dual $\mathfrak{g}^{*}$ of the Lie algebra $\mathfrak{g}$ of $G$, giving the classical phase space as the cotangent bundle $T^{*} G \cong G \times \mathfrak{g}^{*}$. The classical algebra of observables is 
thus the Poisson algebra $\mathcal{P}_{G}=\left(C^{\infty}\left(T^{*} G\right),\{\cdot, \cdot\}, \cdot\right)$, such that ${ }^{1}$

$$
\{f, g\} \equiv \frac{\partial f}{\partial X_{i}} \mathcal{L}_{i} g-\mathcal{L}_{i} f \frac{\partial g}{\partial X_{i}}+c_{i j}^{k} \frac{\partial f}{\partial X_{i}} \frac{\partial g}{\partial X_{j}} X_{k},
$$

for functions $f, g \in C^{\infty}\left(T^{*} G\right)$, Euclidean coordinates $X_{i}$ on $\mathfrak{g}^{*} \cong \mathbb{R}^{d}(d:=\operatorname{dim}(G)) ; \mathcal{L}_{i}$ are Lie derivatives on $G$ (with respect an orthonormal basis of right-invariant vector fields), and $c_{i j}{ }^{k}$ are the structure constants of the Lie algebra $\mathfrak{g}\left(\cong \mathfrak{g}^{*}\right)$ with $i, j, k=1, \ldots, d$ (summation over repeated indexes is assumed).

The quantization of a maximal subalgebra $\mathcal{A}$ of $\mathcal{P}_{G}$ (for which the quantization is consistent) amounts to the definition of an abstract operator ${ }^{*}$-algebra $\mathfrak{U}$, obtained from the classical Poisson algebra. The quantization is defined through a quantization map $\mathcal{Q}: \mathcal{A} \rightarrow \mathcal{U}$. The required properties of the quantization map are as follows. First, $\mathcal{Q}(f)=: \hat{f}$ for all $f \in \mathcal{A}_{G} \subset C^{\infty}(G), \mathcal{A}_{G}$ being the subalgebra of $\mathcal{A} \subset C^{\infty}\left(G \times \mathfrak{g}^{*}\right)$ constant in the second argument, and $\mathcal{Q}\left(X_{i}\right)=: \hat{X}_{i}$, satisfying

$$
[\hat{f}, \hat{g}]=0, \quad\left[\hat{X}_{i}, \hat{f}\right]=i \widehat{\mathcal{L}_{i} f} \in \mathfrak{U}_{G}, \quad\left[\hat{X}_{i}, \hat{X}_{j}\right]=i c_{i j}{ }^{k} \hat{X}_{k},
$$

for all $\hat{f}, \hat{g} \in \mathfrak{U}_{G}$ with $\mathfrak{U}_{G}=\mathcal{Q}\left(\mathcal{A}_{G}\right)$. Second, when restricted to functions $\mathcal{A}_{\mathfrak{g}^{*}} \subset C^{\infty}\left(G \times \mathfrak{g}^{*}\right)$ constant in the first factor, $\mathcal{Q}$ maps to a completion of the universal enveloping algebra of $\mathfrak{g}, \mathfrak{U}_{\mathfrak{g}^{*}}:=\mathcal{Q}\left(\mathcal{A}_{\mathfrak{g}^{*}}\right) \cong \overline{U(\mathfrak{g})}$. If we restrict to the space of polynomials in $\mathfrak{g}^{*}$, then $\mathcal{Q}$ encodes the operator ambiguity coming from the noncommutativity of the elements $\hat{X}_{i}$. The algebra $\mathcal{U}$ generated by $\hat{f}$ and $\hat{X}_{i}$ is then called the "quantum (observable) algebra" for $T^{*} G$.

$\mathfrak{U}_{\mathfrak{g}^{*}}$ is endowed with a natural Hopf algebra structure inherited from the universal enveloping algebra $U$, with coproduct $\Delta_{\mathfrak{g}^{*}}$, counit $\epsilon_{\mathfrak{g}^{*}}$ and antipode $S_{\mathfrak{g}^{*}}$

$$
\begin{aligned}
\Delta_{\mathfrak{g}^{*}}\left(\hat{X}_{i}\right) & =\hat{X}_{i} \otimes \mathbb{1}+\mathbb{1} \otimes \hat{X}_{i}, \\
\epsilon_{\mathfrak{g}^{*}}\left(\hat{X}_{i}\right) & =0, \quad S_{\mathfrak{g}^{*}}\left(\hat{X}_{i}\right)=-\hat{X}_{i} .
\end{aligned}
$$

An Hopf algebra structure in $\mathfrak{U}_{G}$ can be obtained ${ }^{2}$ for exponential Lie groups by considering canonical coordinates (of the first kind) $k: G \rightarrow \mathfrak{g} \cong \mathbb{R}^{d}$ defined through

\footnotetext{
${ }^{1}$ See, e.g., [23] for a general discussion of Poisson structures on cotangent bundles. While there are in general other possible Poisson structures for $T^{*} G$, we here stick to the form (1), which is compatible with the description of the phase space $T^{*} G$ complying with the semidirect product on $G \times \mathfrak{g}^{*}$ implementing the (coadjoint) action of $G$ on $\mathfrak{g}^{*}$. The explicit expression (1) for the semidirect product $T^{*} G \cong G \times \mathfrak{g}^{*}$ can be found in [24].

${ }^{2}$ This construction of the coproduct for exponential Lie groups was introduced in [15].
}

the logarithm map, $k(g)=-i \ln (g)$, setting for the corresponding operators $\epsilon_{G}\left(\hat{k}^{i}\right)=0, S_{G}\left(\hat{k}^{i}\right)=-\hat{k}^{i}$ and

$\Delta_{G}\left(\hat{k}^{i}\right)=\sum_{n=1}^{\infty} \sum_{\substack{k, l \in \mathbb{N} \\ k+l=n}} B_{p_{1} \cdots p_{k} q_{1} \cdots q_{l}} \hat{k}^{p_{1}} \cdots \hat{k}^{p_{k}} \otimes \hat{k}^{q_{1}} \cdots \hat{k}^{q_{k}}$,

where $B_{p_{1} \cdots p_{k} q_{1} \cdots q_{l}} \in \mathbb{R}$ are the coefficient of the Taylor expansion for the Baker-Campbell-Hausdorff formula $k^{i}(g h)=\mathcal{B}(k(g), k(h))^{i}$. Defining the lift of $f \in \mathcal{A}_{G}$ onto the Lie algebra as $f_{k}(k) \equiv f\left(e^{i k}\right)$, the coproduct can be extended to the corresponding $\hat{f} \in \mathfrak{U}_{G}$ as $\Delta_{G}(\hat{f}) \equiv$ $f_{k}\left(\Delta_{G}\left(\hat{k}^{i}\right)\right)$. These definitions satisfy the requirements for the coproduct to be induced by the group multiplication as $\Delta(f)(g, h)=f(g h)$, as well as the group unit and inverse to induce counit and antipode, $\epsilon(f)=f(e)$, $S(f)(g)=f\left(g^{-1}\right)$.

\section{B. The group and algebra representations}

In order to get a concrete realization of the abstract observable algebra $\mathfrak{U}$, one has to construct its representations, in which the elements of the abstract algebra become operators acting on specific Hilbert spaces.

For the system at hand, one can immediately define a "group representation" $\pi_{G}$ of $\mathfrak{U}$ on the Hilbert space $L^{2}(G)$ of square-integrable functions on $G$ with respect to the Haar measure $d g$. This is done by diagonalizing all the operators $\hat{f} \in \mathfrak{U}_{G}$ and setting the action of the $\hat{X}_{i}$ to be given by the Lie derivatives, so that

$$
\begin{aligned}
\left(\pi_{G}(\hat{f}) \psi\right)(g) & \equiv f(g) \psi(g), \\
\left(\pi_{G}\left(\hat{X}_{i}\right) \psi\right)(g) & \equiv i \mathcal{L}_{i} \psi(g),
\end{aligned}
$$

for all $\psi \in C_{c}^{\infty}(G)$ where we restrict ${ }^{3}$ the domain of $\pi_{G}$ to the sbspace of $\mathcal{A}_{G}$ of smooth compactly supported functions $C_{c}(G)$ [dense in $L^{2}(G)$ ] on $G$. The inner product for $\psi, \psi^{\prime} \in L^{2}(G)$ is defined as usual as

$$
\left\langle\psi, \psi^{\prime}\right\rangle_{G} \equiv \int_{G} d g \overline{\psi(g)} \psi^{\prime}(g) .
$$

That (5) is a representation of the quantum algebra (2) is ensured by the fact that the Lie derivative satisfies the Leibniz rule respect to the pointwise product of functions. Such property can be expressed as a compatibility condition between the coproduct $\Delta_{\mathfrak{g}^{*}}$ of $\mathfrak{U}_{\mathfrak{g}^{*}}$ and the pointwise product $m_{G}: f \otimes f^{\prime} \rightarrow f \cdot f^{\prime}$ for $f, f^{\prime} \in C^{\infty}(G)$. More in

\footnotetext{
${ }^{3}$ This is necessary to ensure that $f \psi$ lies in $C_{c}(G)$ for all $\psi \in C_{c}^{\infty}(G)$. In general, since we are dealing with unbounded operators, for their representation on a Hilbert space $\mathcal{H}$, their domain of definition must be restricted to some dense subspaces of $\mathcal{H}$ such that their images under the action of the operators are contained in $\mathcal{H}$.
} 
general if $\pi$ is a representation of $\mathfrak{U}$ on a space $\mathfrak{F}_{m}$ with multiplication $m: f \otimes f^{\prime} \rightarrow f \cdot{ }_{m} f^{\prime}$, the compatibility with the coproduct $\Delta$ can be expressed, for an operator $\hat{T}$ in $\mathcal{U}$, by the commutative diagram

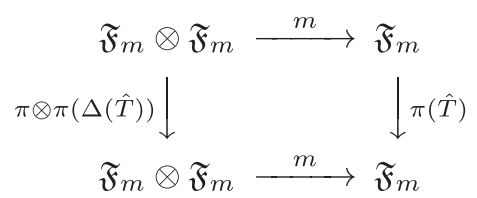

expressing the identity $\pi(\hat{T}) \circ m=m \circ(\pi \otimes \pi)(\Delta(\hat{T}))$.

Besides the group representation $\pi_{G}$, a representation based on functions $\phi(X)$ of coordinates on $\mathfrak{g}^{*}$ (thus analogous of standard functions on momentum space) can also be defined: "the algebra representation". The underlying noncommutativity of the Lie algebra has translate into an appropriately defined noncommutativity of the functions $\phi(X)$. This is done by the introduction of a $\star$-product compatible, in the sense of diagram (7), with the coproduct $\Delta_{G}$ of the algebra of operators $\hat{z}^{i}$ corresponding to a specific parametrization of $G$. As we will see, the required $\star$-product can be defined directly from the choice of quantization map $\mathcal{Q}$.

More precisely, one takes coordinates $z: G \rightarrow \mathfrak{g} \cong \mathbb{R}^{d}$ on $G$ satisfying $z_{k}^{i}(\overrightarrow{0})=0, \frac{\partial}{\partial k^{j}} z_{k}^{i}(\overrightarrow{0})=\delta_{j}^{i}$, where $k$ are canonical coordinates. The coordinates operators $\hat{z}^{i}$ are defined by imposing $\hat{f} \stackrel{!}{=} f_{z}\left(\hat{z}^{i}\right)$, where $f_{z} \circ \vec{z} \equiv f$ for all $f \in C^{\infty}(G)$. The algebra representation $\pi_{\mathfrak{g}}$ then is defined by the actions

$$
\begin{gathered}
\left(\pi_{\mathfrak{g}^{*}}\left(\hat{X}_{i}\right) \phi\right)(X) \equiv X_{i} \star \phi(X), \\
\left(\pi_{\mathfrak{g}^{*}}\left(\hat{z}^{i}\right) \phi\right)(X) \equiv-i \partial^{i} \phi(X),
\end{gathered}
$$

where the second equation means that

$$
\left(\pi_{\mathfrak{g}^{*}}(\hat{f}) \phi\right)(X)=f_{k}(-i \vec{\partial}) \phi(X)
$$

where $f_{k}(k):=f\left(e^{i k}\right) \in C^{\infty}(\mathfrak{g})$ for all $f \in C^{\infty}(G)$. The algebra representation is thus defined on the function space $L_{\star}^{2}\left(\mathfrak{g}^{*}\right) \ni \phi(X)$, which denotes the quotient $C_{c}^{\infty}\left(\mathfrak{g}^{*}\right) / \mathcal{N}$ of the space of smooth compactly supported functions (see footnote 3 ) on $\mathfrak{g}^{*}$ by the subspace $\mathcal{N}$, such that the inner product respect to the Lebesgue measure $d^{d} X$ on $\mathfrak{g}^{*}$

$$
\left\langle\phi, \phi^{\prime}\right\rangle_{\mathfrak{g}^{*}}:=\int_{\mathfrak{g}^{*}} \frac{d^{d} X}{(2 \pi)^{d}}\left(\bar{\phi} \star \phi^{\prime}\right)(X)
$$

is non degenerate, i.e., with $\mathcal{N}:=\left\{\phi \in C^{\infty}\left(\mathfrak{g}^{*}\right):\left\langle\phi, \phi^{\prime}\right\rangle_{\mathfrak{g}^{*}}=0\right\}$. The first equation in (8) is actually a consequence of a stronger condition that one imposes on the $\star$-product:

$$
\left(\pi_{\mathfrak{g}^{*}}\left(f\left(\hat{X}_{i}\right)\right) \phi(X)\right) \equiv f_{\star}(X) \star \phi(X),
$$

for all $f_{\star} \in \mathcal{A}_{\mathfrak{g}^{*}} \subset C^{\infty}\left(\mathfrak{g}^{*}\right)$ such that $f\left(\hat{X}_{i}\right)=\mathcal{Q}\left(f_{\star}\right) \in \mathfrak{U}_{\mathfrak{g}^{*}}$. With this definition the $\star$-product and quantization map are related, as in the deformation quantization framework, by

$$
f_{\star} \star f_{\star}^{\prime}=\mathcal{Q}^{-1}\left(\mathcal{Q}\left(f_{\star}\right) \mathcal{Q}\left(f_{\star}^{\prime}\right)\right) .
$$

As anticipated, the choice of quantization map determines uniquely the $\star$-product to be used in constructing the algebra representation.

The faithfulness of the representation (8) is then guaranteed by noticing that the $\star$-product is such that the observables depending on $\hat{X}_{i}$ are represented through an algebra isomorphism, while $f \rightarrow \pi_{\mathfrak{g}^{*}}(\hat{f})$ is a homomorphism due to commutativity of $\partial^{i}$ on $\mathfrak{g}^{*}$. Finally, one can show (see [15]) that the commutator $\left[\hat{X}_{i}, \hat{z}^{j}\right]=i \widehat{\mathcal{L}_{i} z^{j}}$ is reproduced if the $\star$-product satisfies the property encoded in the commutative diagram (7), i.e., if

$$
\pi_{\mathfrak{g}^{*}}\left(\hat{z}^{i}\right) \circ m_{\mathfrak{g}^{*}}=m_{\mathfrak{g}^{*}} \circ\left(\pi_{\mathfrak{g}^{*}} \otimes \pi_{\mathfrak{g}^{*}}\right)\left(\Delta_{G}\left(\hat{z}^{i}\right)\right) .
$$

Thus, the compatibility condition (7) can also be understood as expressing the compatibility between the choice of quantization map, i.e., of $\star$-product, and the choice of coordinates on the group used in defining the quantum algebra $\mathfrak{U}$. For more details on the algebra representations, see [15].

\section{The noncommutative Fourier transform}

The noncommutative Fourier transform $\mathcal{F}: L^{2}(G) \rightarrow$ $L_{\star}^{2}\left(g^{*}\right)$ [and its inverse $\mathcal{F}^{-1}: L_{\star}^{2}\left(g^{*}\right) \rightarrow L^{2}(G)$ ] is the intertwiner between the group and algebra representation (and vice versa), ensuring their unitary equivalence.

It is expressed as the integral transform:

$$
\begin{aligned}
\tilde{\psi}(X) & :=\mathcal{F}(\psi)(X)=\int_{G} d g E_{g}(X) \psi(g) \in L_{\star}^{2}\left(\mathfrak{g}^{*}\right), \\
\psi(g) & :=\mathcal{F}^{-1}(\tilde{\psi})(g)=\int_{\mathfrak{g}^{*}} \frac{d^{d} X}{(2 \pi)^{d}} \overline{E_{g}(X)} \star \tilde{\psi}(X) \in L^{2}(G) .
\end{aligned}
$$

The kernel of the transformation $E_{g}(X)$ is called the noncommutative plane wave. Its defining equations are identified by requiring that the intertwined function spaces define a representation of the same quantum algebra, and applying the action of $\mathfrak{U}$ in the different representation. The explicit form of $E_{g}(X)$, and its existence, depends then again on the choice of a quantization map, and thus, in turn, on the choice of a deformation quantization $\star$-product.

The intertwining property can be expressed generally as $\mathcal{F} \circ \pi_{G}(\hat{T})=\pi_{\mathfrak{g}^{*}}(\hat{T}) \circ \mathcal{F}$, where $\hat{T} \in \mathcal{U}$. Applying it to the operators $\hat{X}_{i}$ and $\hat{\zeta}^{i}$ respectively, one obtains the following conditions to be satisfied by the Kernel $E_{g}(X)$ : 


$$
\begin{aligned}
& -i \mathcal{L}_{i} E_{g}(X)=X_{i} \star E_{g}(X), \\
& -i \partial^{i} E_{g}(X)=z^{i}(g) E_{g}(X),
\end{aligned}
$$

which can be integrated (modulo the nontrivial global properties of $G$ ) as

$$
\begin{aligned}
E(h g, X) & =e^{k(h) \cdot \overrightarrow{\mathcal{L}}} E(g, X)=e_{\star}^{i k(h) \cdot X} \star E(g, X), \\
E(g, X+Y) & =e^{Y \cdot \vec{\partial}} E(g, X)=e^{i \zeta(g) \cdot Y} E(g, X),
\end{aligned}
$$

where $k(h)=-i \ln (h) \in \mathfrak{g}$ are canonical coordinates, and

$$
e_{\star}^{f(X)}=\sum_{n=0}^{\infty} \frac{1}{n !} \underbrace{f \star \cdots \star f}_{n \text { times }}(X)
$$

Combining the two expressions one can show that the plane wave is given by the $\star$-exponential in terms of the canonical coordinates on the group manifold

$$
\begin{aligned}
E_{g}(X) & =e_{\star}^{i k(g) \cdot X} \\
& =\sum_{n=0}^{\infty} \frac{i^{n}}{n !} k^{j_{1}}(g) \cdots k^{j_{n}}(g) X_{j_{1}} \star \cdots \star X_{j_{n}} .
\end{aligned}
$$

The existence and explicit form of the plane wave (18) is subject to the possible nontrivial global properties of $G$. For the definition of the Fourier transform, since $E(g, X)$ has to be considered only under integration, weak exponentiality ${ }^{4}$ of $G$ is a sufficient condition. Moreover, in case $G$ has compact subgroups, the logarithm map is multivalued, and the definition in terms of canonical coordinates has to refer only to one of the charts covering the group manifold, and extended appropriately beyond it (this can be done in several ways, see [15] and references cited there). Let us discuss this point a little further, briefly.

From the relation (12) it follows that the product of plane waves is given by

$$
\begin{aligned}
E_{g}(X) \star E_{h}(X) & =\mathcal{Q}^{-1}\left(e^{i k(g) \cdot \hat{X}} e^{i k(h) \cdot \hat{X}}\right) \\
& =e_{\star}^{i \mathcal{B}(k(g), k(h)) \cdot X},
\end{aligned}
$$

where $\mathcal{B}(k(g), k(h))$ is given by the application of the BakerCampbell-Hausdorff (BCH) formula to $k(g), k(h) \in \mathfrak{g}$, and

$$
\mathcal{Q}\left(e_{\star}^{i k \cdot X}\right)=e^{i k \cdot \hat{X}} .
$$

In order to have a one-to-one relation between elements of the set of plane waves $\mathcal{E}:=\left\{e^{i k . \hat{X}}: k \in \mathfrak{g}\right\} \subset \mathfrak{U}_{\mathfrak{g}^{*}}$ and the

\footnotetext{
${ }^{4}$ A group $G$ is said to be exponential if its exponential map $\exp _{G}:(\mathfrak{g}) \rightarrow G$ is onto (surjective), in the sense that $\forall g \in G$, $\exists x \in \mathfrak{g}$ such that $\exp _{G}(x)=g$. A group $G$ is said to be "weakly" exponential $[25,26]$ if $\exp _{G}$ is dense in $G$, in the sense that the closure $\overline{\exp _{G} \mathfrak{g}}$ of $\exp _{G} \mathfrak{g}=\left\{\exp _{G}(x): x \in \mathfrak{g}\right\}$ is equal to $G$. See also the discussion at the beginning of Sec. III A.
}

group $G$, one can define the coordinates $k(g)=-i \ln (g)$ to lie in the principal branch, and amend the $\star$-product by a projection onto the principal branch of the logarithm, $\star \rightarrow \star_{p}$. With this definition the set of plane waves constitutes a representation of $G$ respect to the $\star_{p}$-product, since $^{5}$

$$
E_{g}(X) \star_{p} E_{h}(X)=E_{g h}(X)
$$

We will omit in the following the suffix $p$ and implicitly assume that the projection on the principal branch is implemented.

Thus, in terms of canonical coordinates $k(g)$ on the group, the noncommutative plane waves takes the form of a $\star$-exponential with respect to the $\star$-product corresponding to the chosen quantization map.

However, from the second of Eqs. (16), it follows also that there exists a choice of coordinates $z^{i}(g)$ such that the same star-exponentials take the form of classical exponentials

$$
E_{g}(X)=\mathcal{A}(g) e^{i z(g) \cdot X}
$$

with a multiplicative prefactor $\mathcal{A}(g)=E_{g}(0)$. The choice of such coordinates $z^{i}(g)$ and the corresponding prefactor $\mathcal{A}(g)$ are also determined by the quantization map. For this second form the multivaluedness issue affects the choice of coordinates $z: G \rightarrow \mathfrak{g}$.

Finally, it is possible to show (see [15]) that when the $\star$ product is verified to lead to a noncommutative plane wave of the form (22), i.e., when $E_{g}(X)=e_{\star}^{i k(g) \cdot X}=\mathcal{A}(g) e^{i z(g) \cdot X}$, then the $z$-coordinates satisfy the compatibility equation (13), i.e., they are compatible with the $\star$-product in the sense of the commutative diagram (7). This means that the algebra representation is guaranteed to exist, and to be obtained by noncommutative Fourier transform from the group representation, only if such coordinates can be found.

\section{Summary}

Let us summarize schematically the main elements introduced so far. One starts from the phase space of a system whose configuration space is a Lie group $G$, given by the cotangent bundle $T^{*} G \cong G \times \mathfrak{g}^{*}$, and its Poisson algebra $\mathcal{P}_{G}$. A quantization of (a maximal subalgebra $\mathcal{A}$ of) $\mathcal{P}_{G}$ can be obtained through a quantization map $\mathcal{Q}$, leading to the quantum algebra $\mathfrak{U} \approx \mathfrak{U}_{G} \times \mathfrak{U}_{\mathfrak{g}^{*}}$. The latter is endowed, for each of its subspaces $\mathfrak{U}_{G}$ and $\mathfrak{U}_{\mathbf{g}^{*}}$, with a natural Hopf-algebra structure characterized by $\left(\Delta_{G}, \epsilon_{G}, S_{G}\right)$ and $\left(\Delta_{\mathfrak{g}^{*}}, \epsilon_{\mathfrak{g}^{*}}, S_{\mathfrak{g}^{*}}\right)$.

${ }^{5}$ Without the projection on the principal branch, the coordinate $\mathcal{B}^{i}(k(g), k(h))$ in (19) may lie in any branch of the logarithm. 
Two different representation of $\mathfrak{U}$ can be defined, the group representation $\pi_{G}$ on $L^{2}(G)$, and the algebra representation $\pi_{\mathfrak{g}^{*}}$ on $L_{\star}^{2}\left(\mathfrak{g}^{*}\right)$. The latter is characterized by the definition of a suitable $\star$-product compatible with the quantization map, and determined by it. Both representations satisfy the compatibility condition between their natives product and coproduct, as depicted in the diagram (7), which can then be understood as a condition for the existence of the representation.

The noncommutative Fourier transform $\mathcal{F}: L^{2}(G) \rightarrow$ $L_{\star}^{2}\left(g^{*}\right)$ is the intertwiner between the group and algebra representation, ensuring their unitary equivalence. Its kernel is the noncommutative plane wave $E_{g}(X)$, whose existence and precise expression depend again on the chosen quantization map, and thus of $\star$-product. The explicit form of the plane wave can indeed be written in terms of $\star$-exponentials $E_{g}(X)=\exp _{\star}(k(g) \cdot X)$, where $k(g)=-i \ln (g) \quad$ are canonical coordinates, or as ordinary exponentials (with a prefactor) $E_{g}(X)=\mathcal{A}(z(g)) \exp (z(g) \cdot X)$ in terms of coordinates $z$ such that $z_{k}(0)=0$ and $\frac{\partial}{\partial k^{i}} z_{k}^{j}(0)=\delta_{i}^{j}$ $\left[z_{k}(k)=z\left(e^{i k}\right)\right]$. The existence of these kind of coordinates ensures that the compatibility condition (7) is fulfilled, tying together the existence of the noncommutative Fourier transform with the one of the algebra representation.

\section{THE DUFLO MAP AND LIE ALGEBRA REPRESENTATION FOR THE LORENTZ GROUP}

We will now proceed to constructing the structures introduced in the previous section for the case in which the configuration space of our system is the Lorentz group. We will first look for suitable coordinates in which to define the noncommutative plane waves. As explained, the definition of the plane wave depends on the quantization map, which in turn specifies the $\star$-product structure. We will work the Duflo quantization map [16,27,28], which has already found a number of applications in the quantum gravity context [17-22]. After defining the properties of the Duflo map, we will derive the explicit expression of the Duflo plane wave for the Lorentz group, as a star exponential, and as the ordinary exponential with prefactor in terms of suitable coordinates on the group. We will then discuss the properties of the algebra representation and show explicitly the structure of the star product for the lowest order polynomials.

These structures, in particular the plane waves, will finally allow us to define the noncommutative Fourier transform for the Lorentz group for the Duflo quantization map, in the following Sec. IV.

\section{A. Parametrization of the Lorentz group}

As discussed, the definition of the noncommutative plane wave, and thus of the noncommutative Fourier transform, requires the group to be at least weakly exponential. Exponentiability indeed ensures that the image of the exponential map $\exp : \mathfrak{g} \rightarrow G$ is onto, i.e., that all the points of $G$ are obtained through exponentiation of its Lie algebra. Also, if a group is weakly exponential $[25,26]$ the image of exp : $\mathfrak{g} \rightarrow G$ is dense in $G$. Since the noncommutative Fourier transform is defined only under integration, weak exponentiability is a sufficient condition for its uniqueness, as the canonical coordinates $k(g)=$ $-i \ln (g)$ defining the plane waves will cover the whole group except for a set of points of zero measure. So, in order to define the noncommutative Fourier transform for the Lorentz group we must first discuss the relation between its parametrization and the exponential map (we follow mainly the discussion in [29]).

The (homogeneous) Lorentz group is usually defined by two basic matrix representations: as the group ${ }^{6} \mathrm{SO}(3,1)$ of real orthogonal $4 \times 4$ matrices with unit determinant, preserving the bilinear form of Lorentzian signature $(3,1)$, or as the group $\operatorname{SL}(2, \mathbb{C})$ of complex unimodular $2 \times 2$ matrices. As topological groups, $\operatorname{SL}(2, \mathbb{C})$ is the double covering of $\mathrm{SO}(3,1)$. Moreover since $\operatorname{SL}(2, \mathbb{C})$ is simply connected it is also the universal covering of $\mathrm{SO}(3,1)$, while $\mathrm{SO}(3,1)$ is isomorphic to $\mathrm{SL}(2, \mathbb{C}) /\{I,-I\}$, where $I$ and $-I$ are the central elements of $\operatorname{SL}(2, \mathbb{C})$, and thus $\operatorname{SO}(3,1)$ is doublyconnected.

While for a compact connected (matrix) group one can prove that the exponential map is surjective [30], when the group is noncompact, as for $\mathrm{SO}(3,1)$ or $\mathrm{SL}(2, \mathbb{C})$, surjectiveness is not guaranteed. In particular one can show that $\mathrm{SO}(3,1)$ is exponential, while $\operatorname{SL}(2, \mathbb{C})$ is not [31], i.e., not all the points of $\operatorname{SL}(2, \mathbb{C})$ are in the image of the exponential map applied to its Lie algebra $\mathfrak{I l}(2, \mathbb{C})$. However, a complex connected Lie group, as is the case of $\operatorname{SL}(2, \mathbb{C}$ ), is always weakly exponential (see for instance [32]), so that the image of $\exp : \mathfrak{g l}(2, \mathbb{C}) \rightarrow \operatorname{SL}(2, \mathbb{C})$ is dense. As we stressed, this is all that is needed for a welldefined noncommutative Fourier transform and algebra representation.

An element of $\operatorname{SL}(2, \mathbb{C})$ can be parametrized as

$$
a=a_{0} \mathbb{1}_{2}+\vec{a} \cdot \vec{\sigma},
$$

where $\mathbb{1}_{2}$ is the identity matrix in 2 dimensions, $a_{0}, a_{j}$, with $j=1,2,3$, are complex numbers, and $\sigma_{j}$ are Pauli matrices (Hermitian traceless $2 \times 2$ matrices)

$\sigma_{1}=\left(\begin{array}{ll}0 & 1 \\ 1 & 0\end{array}\right), \quad \sigma_{2}=\left(\begin{array}{cc}0 & -i \\ i & 0\end{array}\right), \quad \sigma_{3}=\left(\begin{array}{cc}1 & 0 \\ 0 & -1\end{array}\right)$,

satisfying the Lie brackets

\footnotetext{
${ }^{6} \mathrm{We}$ restrict in this manuscript to the so-called proper orthocronous Lorentz group, the identity component of $\mathrm{O}(3,1)$, consisting of the set of Lorentz transformations that preserve the orientation of "spatial" and "temporal" dimensions.
} 


$$
\left[\sigma_{i}, \sigma_{j}\right]=2 i \epsilon_{i j k} \sigma_{k}
$$

The conjugacy classes of $\operatorname{SL}(2, \mathbb{C})$ are instead represented by the matrices $[31,32]$

$$
\left(\begin{array}{cc}
\alpha & 0 \\
0 & \alpha^{-1}
\end{array}\right), \quad\left(\begin{array}{ll}
1 & 1 \\
0 & 1
\end{array}\right), \quad\left(\begin{array}{cc}
-1 & 1 \\
0 & -1
\end{array}\right) .
$$

Canonical coordinates (of the first kind) on $\operatorname{SL}(2, \mathbb{C})$ are defined $[30,31]$ by applying the exponential map to its Lie algebra $\mathfrak{g l}(2, C)$. One way of realizing this is as the complexification of $\mathrm{SU}(2)$ by means of a complex rotation vector [29]

$$
\zeta_{i}=\rho_{i}+i \beta_{i}, \quad i=1,2,3
$$

where $\rho_{i}, \beta_{i}$ are real parameters, so that, choosing the basis of $\mathfrak{s l}(2, C)$ represented by Pauli matrices, an element of $\operatorname{SL}(2, \mathbb{C})$ is written as

$$
a=\exp \left(\frac{i}{2} \vec{\zeta} \cdot \vec{\sigma}\right)
$$

With this definition one gets the relations

$a_{0}=\cos \left(\frac{1}{2}(\phi+i \eta)\right) \quad a_{j}=i \frac{\sin \left(\frac{1}{2}(\phi+i \eta)\right)}{\phi+i \eta} \zeta_{j}$,

where we define the complex rotation angle $\phi+i \eta$, with $\eta \geq 0$, related to $\vec{\zeta}$ by (denoting $x^{2}=\vec{x} \cdot \vec{x}$ )

$$
(\phi+i \eta)^{2}=\zeta^{2}, \quad \phi^{2}-\eta^{2}=\rho^{2}-\beta^{2}, \quad \phi \eta=\vec{\rho} \cdot \vec{\beta} .
$$

The meaning of $\phi$ and $\eta$ can be better understood considering the action of a $\operatorname{SL}(2, \mathbb{C})$ matrix $(23)$ on a point in Minkowski spacetime $x=\left(x^{0}, x^{1}, x^{2}, x^{3}\right)$ defined as

$$
\boldsymbol{x}=x^{0} \mathbb{1}+\vec{x} \cdot \vec{\sigma}=\left(\begin{array}{cc}
x^{0}+x^{3} & x^{1}-i x^{2} \\
x^{1}+i x^{2} & x^{0}-x^{3}
\end{array}\right),
$$

so that

$$
\boldsymbol{x}^{\prime}=a \boldsymbol{x} a^{\dagger} \rightarrow x^{\prime}=\Lambda(a) x .
$$

The transformation matrices $\Lambda(a)$ form the elements of the group $\mathrm{SO}(3,1)$. This is identical to the group of linear transformations leaving invariant the metric $\eta=\operatorname{diag}\{1,-1,-1,-1\}$, described by $4 \times 4$ unimodular orthogonal matrices generated by the set of (purely imaginary traceless) matrices defined by

$$
\left(M_{a b}\right)_{k l}=i\left(\eta_{a k} \delta_{b l}-\eta_{a l} \delta_{b k}\right), \quad a, b, k, l=0,1,2,3,4
$$

with commutation rules
$\left[M_{a b}, M_{c d}\right]=i\left(\eta_{a d} M_{b c}+\eta_{b c} M_{a d}-\eta_{a c} M_{b d}-\eta_{b d} M_{a c}\right)$,

so that an element of $\mathrm{SO}(3,1)$ is (repeated indexes are summed, throughout the manuscript)

$$
\Lambda(\alpha)=\exp \left(\frac{i}{2} \alpha_{i j} M_{i j}\right)
$$

Defining the generators

$J_{i}=\frac{1}{2} \epsilon_{i j k} M_{j k}, \quad N_{i}=M_{0 i}, \quad i, j=1,2,3$,

corresponding to rotation and boost transformations, satisfying the commutation rules

$$
\begin{aligned}
{\left[J_{i}, J_{j}\right] } & =i \epsilon_{i j k} J_{k}, \quad\left[J_{i}, N_{j}\right]=i \epsilon_{i j k} N_{k}, \\
{\left[N_{i}, N_{j}\right] } & =-i \epsilon_{i j k} J_{k},
\end{aligned}
$$

we can express an element of the group as

$$
\Lambda(\vec{\rho}, \vec{\beta})=\exp \{i \vec{\rho} \cdot \vec{J}+i \vec{\beta} \cdot \vec{N}\}
$$

where

$$
\rho_{i}=\frac{1}{2} \epsilon_{i j k} \alpha_{j k}, \quad \beta_{i}=\alpha_{0 i}
$$

The explicit expression of $\Lambda(\vec{\rho}, \vec{\beta})$ is given in the Appendix A 1, where the relation between $\mathrm{SO}(3,1)$ and $\operatorname{SL}(2, \mathbb{C})$ is also discussed. It is important to notice that the parameters domain is different for the two groups: the multivaluedness of the logarithmic map (the inverse of the exponential map) results from the periodicity of the compact subgroup of rotations. As shown in Appendix A 1, analogously to the relation between $\mathrm{SO}(3)$ and $\mathrm{SU}(2)$, $\operatorname{SL}(2, \mathbb{C})$ "covers twice" $\mathrm{SO}(3,1)$, with the isomorphism $\operatorname{SO}(3,1) \simeq \operatorname{SL}(2, \mathbb{C}) /\{\mathbb{1},-\mathbb{1}\}$.

In order to show the weak exponentiability of the Lorentz group, one can study the behavior of the logarithm map by inverting relation (28). The study of the branch points for the logarithmic map for the Lorentz group is performed in Appendix A 2. Restricting it to its principal values, the complex rotation vector is holomorphic in $a_{0}$ except for a branch cut extending on the real axis of $a_{0}$ from -1 to $-\infty$. Thus, except for the branch cut, the canonical coordinates provided by the exponential map, represented by the complex rotation vector $\vec{\zeta}=\vec{\rho}+i \vec{\beta}$, are holomorphic functions parametrizing the whole $\operatorname{SL}(2, \mathbb{C})$ group. Accordingly, the corresponding range of values of $\phi$ for which the complex rotation vector is single-valued is $\phi \in(-2 \pi, 2 \pi]$ for $\operatorname{SL}(2, \mathbb{C})$ and $\phi \in(-\pi, \pi]$ for $\operatorname{SO}(3,1)$. 
This defines the principal branch respectively for the $\mathrm{SL}(2, \mathbb{C})$ and $\mathrm{SO}(3,1)$ parametrizations.

Thus, in the following, we consider a generic element of the Lorentz group in its exponential representation (38) or (28)

$$
g=\exp \{i \vec{\rho} \cdot \vec{J}+i \vec{\beta} \cdot \vec{N}\}
$$

where the generators $J_{i}$ and $N_{i}$ are the ones defined in this section, and, depending on the chosen group representation, can be considered to be the generators (36) of $\mathrm{SO}(3,1)$, $J_{i}=\frac{1}{2} \epsilon_{i j k} M_{j k}$ and $N_{i}=M_{0 i}$, or the generators $J_{i} \equiv \frac{1}{2} \sigma_{i}$ and $N_{i} \equiv \frac{1}{2} i \sigma_{i}$ of the real ${ }^{7}$ Lie algebra $\mathfrak{g} \mathfrak{l}(2, \mathbb{C})_{R}$, satisfying in both cases the Lie brackets (37), the distinction between the two groups being given by the domain of the "rotation" parameter $\phi$ as discussed above. An element of the Lie algebra is then $x=x^{i} e_{i} \in \mathfrak{g}$, with $e_{i}$ the basis of generators $e_{i} \equiv\left(J_{i}, N_{i}\right)$, and $x^{i}$ the set of canonical coordinates $k^{i} \equiv\left(\rho^{i}, \beta^{i}\right)$.

\section{B. The Duflo quantization map for the Lorentz group and the noncommutative plane waves}

The Duflo map was introduced in [16] as a generalization to arbitrary finite-dimensional Lie algebras of the Harish-Chandra isomorphism between invariant polynomials on the dual of a Lie algebra and the center of the corresponding universal enveloping algebra. The isomorphism was proved in a different form within the formalism of deformation quantization of general Poisson manifolds [27] (see also [28]), and used then in different contexts in the quantum gravity literature [17-22,33]. An important property of the Duflo map is that it realizes the isomorphism between the set $\operatorname{Sym}(\mathfrak{g})^{\mathfrak{g}}$ of invariant polynomials under the (adjoint) action of $G$, and the set $U(\mathfrak{g})^{\mathfrak{g}}$ of $G$-invariant differential operators on the enveloping algebra, corresponding to the center of the enveloping algebra, $U(\mathfrak{g})^{\mathfrak{g}}=\mathcal{Z}(U(\mathfrak{g}))$. Thus, invariant polynomials $\operatorname{Sym}(\mathfrak{g})^{\mathfrak{g}}$ map to Casimirs of the quantum algebra, and the map preserves the algebra structure of such polynomials, so the Duflo quantization map $\mathcal{D}$ has the remarkable property that for two elements $\alpha$ and $\beta$ of $U(\mathfrak{g})^{\mathfrak{g}}, \mathcal{D}(\alpha) \mathcal{D}(\beta)=\mathcal{D}(\alpha \beta)$. We point out that while the Duflo map is uniquely determined by the isomorphism property only on the invariant subspace $\operatorname{Sym}(\mathfrak{g})^{\mathfrak{g}}$ of $\operatorname{Sym}(\mathfrak{g})$, we will extend it to all of $\operatorname{Sym}(\mathfrak{g})$ in a natural way. ${ }^{8}$

Concretely, the Duflo map is defined by the composition

$$
\mathcal{D}=\mathcal{S} \circ j^{\frac{1}{2}}(\partial)
$$

\footnotetext{
${ }^{7} \mathfrak{g l}(2, \mathbb{C})$ can be considered a real Lie algebra generated of dimension 6 with basis vectors $\left\{\sigma_{i}\right\},\left\{i \sigma_{i}\right\}$, not to be confused with its real forms $\mathfrak{G} \mathfrak{t}(2)$ or $\mathfrak{g l}(2, \mathbb{R})$.

${ }^{8} \mathrm{We}$ refer to [15] for the definition of the symmetric algebra $\operatorname{Sym}(\mathfrak{g})$ and the universal enveloping algebra $U(\mathfrak{g})$.
}

of the "symmetrization map" $S$,

$$
\mathcal{S}\left(X_{i_{1}} \cdots X_{i_{n}}\right)=\frac{1}{n !} \sum_{\sigma \in S_{n}} \hat{X}_{i_{\sigma_{1}}} \cdots \hat{X}_{i_{\sigma_{n}}}
$$

with $S_{n}$ is the symmetric group of order $n$, with the function on the Lie algebra $\mathfrak{g}$

$$
j(x)=\operatorname{det}\left(\frac{\sinh \frac{1}{2} \operatorname{ad}_{x}}{\frac{1}{2} \operatorname{ad}_{x}}\right), \quad x \in \mathfrak{g},
$$

where in (41) one substitutes $x^{i}$ with $\partial^{i}=\partial / \partial X_{i}$ where $X \in \mathfrak{g}^{*}$. Here $\operatorname{ad}_{x}$ is in the adjoint representation of $\mathfrak{g}$. Notice also that with this definition the Duflo function (43) coincides with the Jacobian of the exponential map [34,35], i.e., for $g=\exp (x)$

$$
d g=j(x) d x
$$

As we have explained in Sec. II, once we have chosen the Duflo quantization map, the compatibility between the associated $\star$-product, given by relation (12) as

$$
f_{\star} \star f_{\star}^{\prime}=\mathcal{D}^{-1}\left(\mathcal{D}\left(f_{\star}\right) \mathcal{D}\left(f_{\star}^{\prime}\right)\right),
$$

and the coproduct on the algebra of functions on the group, and thus the existence of the algebra representation, is guaranteed by the construction of the noncommutative plane wave, the kernel of the transformation between the group and algebra representations. Our next goal is then to derive the explicit expression of this noncommutative plane wave, and in particular its expression as a standard exponential (with a certain prefactor) in terms of specific coordinates on the group.

We need first to calculate the function $j(x)$ for the Lorentz group. We consider a generic element of the Lorentz group written in its exponential representation (38) or (28),

$$
g=\exp \{i \vec{\rho} \cdot \vec{J}+i \vec{\beta} \cdot \vec{N}\}
$$

where the generators $J_{i}$ and $N_{i}$ are the ones defined in the previous section, and can be considered to correspond to the $J_{i}, N_{i}$ matrices generating $\mathrm{SO}(3,1)$ or to the generators $J_{i} \equiv \frac{1}{2} \sigma_{i}, N_{i} \equiv \frac{1}{2} i \sigma_{i}$ of the real ${ }^{9}$ Lie algebra $\mathfrak{g l}(2, \mathbb{C})_{R}$, in both cases satisfying the Lie brackets (37), the distinction between the two groups being given by the domain of the rotation parameter $\phi$ as discussed in the previous section. The calculation of the Duflo function $j(x)$ on the element (40) is performed in Appendix B 1, and, for an element of the Lie algebra $x=x_{J}^{i} J_{i}+x_{N}^{i} N_{i}$, gives

\footnotetext{
${ }^{9} \mathfrak{g l}(2, \mathbb{C})$ can be considered to be a real Lie algebra generated of dimension 6 with basis vectors $\left\{\sigma_{i}\right\},\left\{i \sigma_{i}\right\}$, not to be confused with one of its real forms $\mathfrak{g} \mathfrak{i t}(2)$ or $\mathfrak{g l}(2, \mathbb{R})$.
} 


$$
j^{\frac{1}{2}}(x)=4 \frac{\left|\sinh \left(\frac{1}{2} x_{\zeta}\right)\right|^{2}}{\left|x_{\zeta}^{2}\right|},
$$

where $x_{\zeta}=\sqrt{\vec{x}_{\zeta} \cdot \vec{x}_{\zeta}}$ and $x_{\zeta}^{i}=x_{J}^{i}+i x_{N}^{i}$. We can now apply the Duflo function $j^{\frac{1}{2}}(\partial)$ on exponentials $\exp \left(i k^{i} X_{i}\right)=\exp \left(i \rho^{i} X_{i}^{J}+i \beta^{i} X_{i}^{N}\right)$, with coordinates $k^{i} \equiv$ $\left(\rho^{i}, \beta^{i}\right)$ corresponding to canonical coordinates on the group $k(g)=-i \ln (g)$, and coordinates on $\mathfrak{g}^{*} X_{i} \equiv$ $\left(X_{i}^{J}, X_{i}^{N}\right)$ associated respectively to the $J_{i}$ and $N_{i}$ generators. ${ }^{10}$ Thus, after the substitution $x_{J}^{i} \rightarrow \partial_{J}^{i}, x_{N}^{i} \rightarrow \partial_{N}^{i}$ (i.e., $x_{\zeta}^{i} \rightarrow \partial_{\zeta}^{i}=\partial_{J}^{i}+i \partial_{N}^{i}$ ), we obtain

$$
\begin{aligned}
& \left(j^{\frac{1}{2}}(\partial) \exp \right)\left(i \vec{\rho} \cdot \vec{X}^{J}+i \vec{\beta} \cdot \vec{X}^{N}\right) \\
& \quad=4 \frac{\left|\sin \left(\frac{1}{2} \zeta\right)\right|^{2}}{\left|\zeta^{2}\right|} e^{i \vec{\rho} \cdot \vec{X}^{J}+i \vec{\beta} \cdot \vec{X}^{N}} \\
& \quad=4 \frac{\cosh ^{2}\left(\frac{1}{2} \eta\right) \sin ^{2}\left(\frac{1}{2} \phi\right)+\sinh ^{2}\left(\frac{1}{2} \eta\right) \cos ^{2}\left(\frac{1}{2} \phi\right)}{\phi^{2}+\eta^{2}} e^{i \vec{\rho} \cdot \vec{X}^{J}+i \vec{\beta} \cdot \vec{X}^{N}},
\end{aligned}
$$

where $\zeta_{i}=\rho_{i}+i \beta_{i}$, and we have considered also the parametrization (30), $(\phi+i \eta)^{2}=\zeta^{2}, \phi^{2}-\eta^{2}=\rho^{2}-\beta^{2}$, $\phi \eta=\vec{\rho} \cdot \vec{\beta}$.

Notice now that the exponential keeps the same form under the symmetrization map (42):

$$
\mathcal{S}\left(e^{i k^{i} X_{i}}\right)=e^{i k^{i} \hat{X}_{i}}
$$

Using the above results, we finally obtain the action of the Duflo map on the exponential function

$$
\begin{aligned}
\mathcal{D}\left(e^{i \vec{\rho} \cdot \vec{X}^{J}+i \vec{\beta} \cdot \vec{X}^{N}}\right)= & 4 \frac{\cosh ^{2}\left(\frac{1}{2} \eta\right) \sin ^{2}\left(\frac{1}{2} \phi\right)+\sinh ^{2}\left(\frac{1}{2} \eta\right) \cos ^{2}\left(\frac{1}{2} \phi\right)}{\phi^{2}+\eta^{2}} \\
& \times e^{i \vec{p} \cdot \hat{X}^{J}+i \vec{\beta} \cdot \hat{X}^{N}} .
\end{aligned}
$$

The last equation allows us to define the noncommutative plane wave corresponding to the Duflo quantization map for the Lorentz group. This can be given in its expression in terms of $\star$-exponential (18), which in turn encodes the property (45) [as in (20)], and thus defines the $\star$-product for the Duflo map. We have indeed

$$
\mathcal{D}\left(E_{g}(X)\right)=\mathcal{D}\left(e_{\star}^{i \vec{\rho}(g) \cdot \vec{X}^{J}+i \vec{\beta}(g) \cdot \vec{X}^{N}}\right)=e^{i \vec{\rho}(g) \cdot \hat{X}^{J}+i \vec{\beta}(g) \cdot \hat{X}^{N}} .
$$

At the same time the last equation can be inverted, and using Eq. (50) we get the relation between the plane wave as a $\star$-exponential and as a standard exponential with prefactor (22):

\footnotetext{
${ }^{10}$ More precisely $X_{i}$ are the coordinates on the Lie algebra $\mathfrak{g}^{*}$ defined by the basis $\left\{\tilde{e}_{i}\right\}$ dual to $\left\{e_{i}\right\} \equiv\left\{J_{i}, N_{i}\right\}$ with the canonical pairing $\left\langle\tilde{e}_{i}, e_{j}\right\rangle=\delta_{i j}, X=X_{i} \tilde{e}_{i} \in \mathfrak{g}^{*}$.
}

$$
\begin{aligned}
E_{g}(X) & =\mathcal{D}^{-1}\left(e^{i \vec{\rho}(g) \cdot \hat{X}^{J}+i \vec{\beta}(g) \cdot \hat{X}^{N}}\right)=e_{\star}^{i \vec{\rho}(g) \cdot \vec{X}^{J}+i \vec{\beta}(g) \cdot \vec{X}^{N}} \\
& =\mathcal{A}(\vec{\rho}(g), \vec{\beta}(g)) e^{i \vec{\rho}(g) \cdot \vec{X}^{J}+i \vec{\beta}(g) \cdot \vec{X}^{N}}
\end{aligned}
$$

with

$$
\begin{aligned}
& \mathcal{A}(\vec{\rho}(g), \vec{\beta}(g)) \\
& =\frac{\phi(g)^{2}+\eta(g)^{2}}{4\left(\cosh ^{2}\left(\frac{1}{2} \eta(g)\right) \sin ^{2}\left(\frac{1}{2} \phi(g)\right)+\sinh ^{2}\left(\frac{1}{2} \eta(g)\right) \cos ^{2}\left(\frac{1}{2} \phi(g)\right)\right)},
\end{aligned}
$$

where $(\phi, \eta) \equiv(\phi(\vec{\rho}, \vec{\beta}), \eta(\vec{\rho}, \vec{\beta}))$. Expression (52) shows that within the Duflo quantization map the coordinates for which the noncommutative plane wave takes the form of the standard exponential are still the canonical coordinates $z(g)=k(g)=-i \ln (g) \equiv(\vec{\rho}, \vec{\beta})$, but with a prefactor $\mathcal{A}(\vec{\rho}, \vec{\beta})$, amounting, as expected from Eq. (44), to the inverse of the square root of the Jacobian of the exponential map [see Eq. (A19)]. Notice that this is exactly what happens, for example, in the simpler $\mathrm{SU}(2)$ case, studied in [15]. We have thus obtained the explicit form of the plane wave as a standard exponential, ensuring the existence of the algebra representation, which we are now going to discuss.

\section{The noncommutative algebra representation from the Duflo map}

We mentioned in Sec. II, and it was proven in [15], that the coordinates for which the noncommutative plane wave can be written as a standard exponential (plus prefactor), satisfy the compatibility relation (13) (or (7) ) with the $\star$-product, which, in turn, ensures the existence of the algebra representation for this choice of quantization map. In other words, having derived relation (52)-(53), we have ensured that, for the $\star$-product associated to the Duflo map, the action (8) on functions $\phi(X) \in C_{c}^{\infty}\left(\mathfrak{g}_{\star}\right)\left(X_{i} \equiv\left(X_{i}^{J}, X_{i}^{N}\right) z_{i} \equiv\left(\rho_{i}, \beta_{i}\right)\right.$, $\left.\partial^{i}=\partial / \partial X_{i}\right)$

$$
\begin{gathered}
\left(\pi_{\mathfrak{g}^{*}}\left(\hat{X}_{i}\right) \phi\right)(X) \equiv X_{i} \star \phi(X), \\
\left(\pi_{\mathfrak{g}^{*}}\left(\hat{z}^{i}\right) \phi\right)(X) \equiv-i \partial^{i} \phi(X),
\end{gathered}
$$

is a representation of the quantum algebra (2)

$$
\begin{gathered}
{\left[\hat{X}_{i}^{J}, \hat{X}_{j}^{J}\right]=i \epsilon_{i j}^{k} \hat{X}_{k}^{J}, \quad\left[\hat{X}_{i}^{J}, \hat{X}_{j}^{N}\right]=i \epsilon_{i j}^{k} \hat{X}_{k}^{N},} \\
{\left[\hat{X}_{i}^{N}, \hat{X}_{j}^{N}\right]=-i \epsilon_{i j}^{k} \hat{X}_{k}^{N},} \\
{\left[\hat{\rho}_{i}, \hat{\rho}_{j}\right]=\left[\hat{\beta}_{i}, \hat{\beta}_{j}\right]=\left[\hat{\rho}_{i}, \hat{\beta}_{j}\right]=0,} \\
{\left[\hat{X}_{i}, \hat{z}_{j}\right]=i \widehat{\mathcal{L}_{X_{i}} z_{j}} .}
\end{gathered}
$$

While the above statement can be proved in general (see [15]) using the properties of the $\star$-product, we can still show more 
explicitly that this is indeed the case for the structures derived in the previous section. Notice first that relations (56) are trivially satisfied by the representation (54) due to the commutativity of ordinary derivatives. Let us then evaluate the $\star$-product between the lowest order monomials. Considering expressions $(52)^{11}$ the $\star$-product between $n$ coordinates of $\mathfrak{g}^{*}$ can be evaluated through the formula

$$
\begin{aligned}
X_{i_{1}} \star X_{i_{2}} \star \cdots X_{i_{n}}= & \left.(-i)^{n} \frac{\partial^{n}}{\partial k_{1}^{i_{1}} \partial k_{2}^{j_{2}} \cdots \partial k_{3}^{i_{n}}}\right|_{k_{1}=k_{2}=\cdots=k_{n}=0} \\
& \times \mathcal{D}^{-1}\left(e^{i \overrightarrow{\mathcal{B}}\left(k_{1}, k_{2}, \ldots, k_{n}\right) \cdot \hat{X}}\right) .
\end{aligned}
$$

From this formula one obtains (the explicit calculation is reported in Appendix B 2, where third order monomials are also reported for completeness):

$$
\begin{aligned}
& X_{i}^{J} \star X_{j}^{J}=X_{i}^{J} X_{j}^{J}+\frac{i}{2} \epsilon_{i j}^{k} X_{k}^{J}-\frac{1}{6} \delta_{i j}, \\
& X_{i}^{J} \star X_{j}^{N}=X_{i}^{J} X_{j}^{N}+\frac{i}{2} \epsilon_{i j}^{k} X_{k}^{N}, \\
& X_{i}^{N \star X_{j}^{N}}=X_{i}^{N} X_{j}^{N}-\frac{i}{2} \epsilon_{i j}^{k} X_{k}^{J}+\frac{1}{6} \delta_{i j} .
\end{aligned}
$$

These relations show immediately that Eqs. (55) are fulfilled by the representation (54), i.e., that

$$
X_{i} \star X_{j} \star \phi(X)-X_{j} \star X_{i} \star \phi(X)=i \epsilon_{i j}^{k} X_{k}^{J} \star \phi(X) .
$$

Finally we must check the commutators (57). It is enough to consider the action (54) on plane waves (52), since, if the noncommutative Fourier transform can be defined, as we will do in the next section, any function can be decomposed in terms of its plane-wave basis. We need to show then that

$$
\begin{gathered}
-i X_{i} \star \partial^{j} E_{g}(X)+i \partial^{j}\left(X_{i} \star E_{g}(X)\right) \\
=\left.i\left(\mathcal{L}_{X_{i}} z_{j}(g)\right)\right|_{z_{j}(g)=-i \partial^{j}} E_{g}(X) .
\end{gathered}
$$

From relations (15) defining the plane wave we have that the left-hand side (1.h.s.) can be rewritten as

$$
-i z_{j}(g) \mathcal{L}_{X_{i}} E_{g}(X)+\partial^{j}\left(\mathcal{L}_{X_{i}} E_{g}(X)\right)
$$

The Lie derivative

\footnotetext{
${ }^{11}$ Obviously, knowing the definition of the Duflo map (41) and the general relation between the quantization map and the associated $\star$-product (12), the action of any operator in the algebra representation and the explicit expression of any $\star-$ monomial can be computed directly. Having at hand the formula for the exponential elements, however, allows a more immediate calculation.
}

$$
\mathcal{L}_{X_{i}} f(g)=\left.\frac{d}{d t} f\left(e^{i t e_{i}} g\right)\right|_{t=0}
$$

can be expressed in terms of coordinates $z^{i}$ for which $E_{g}(X)=\mathcal{A}(z(g)) \exp (i z(g) \cdot X)$ as

$$
\begin{aligned}
\mathcal{L}_{X_{i}} E_{g}(X) & =L_{i}^{j}(z(g)) \partial_{z_{j}} E_{g}(X) \\
& =L_{i}^{j}(z(g))\left(\partial_{z_{j}} \mathcal{A}(z(g))+i X_{j} \mathcal{A}(z(g))\right) e^{i z(g) \cdot X}
\end{aligned}
$$

so that

$$
\begin{aligned}
\partial^{j}\left(\mathcal{L}_{X_{i}} E_{g}(X)\right)= & i L_{i}^{j}(z(g)) \mathcal{A}(z(g)) e^{i z(g) \cdot X} \\
& +L_{i}^{j}(z(g))\left(\partial_{z_{j}} \mathcal{A}(z(g))+i X_{j} \mathcal{A}(z(g))\right) \\
& \times \partial^{j} e^{i z(g) \cdot X} .
\end{aligned}
$$

We now use the second of relations (15), and the relation $\mathcal{L}_{X_{i}} z_{j}(g)=L_{i}^{j}(z(g))$ to rewrite the last expression as

$$
i\left(\mathcal{L}_{X_{i}} z_{j}(g)\right) E_{g}(X)+i z_{j}(g) \mathcal{L}_{X_{i}} E_{g}(X)
$$

which, substituted in (62), and using again the second of (15), returns (61). Thus, we confirm the relations (54) define a representation of (57). Moreover, as mentioned already, one can show in general (see [15]) that this amounts to the compatibility between (the coproduct for) the coordinates $z_{i}(g)$ and the $\star$-product in the sense of the commutative diagram (7).

\section{THE NONCOMMUTATIVE FOURIER TRANSFORM FOR THE LORENTZ GROUP}

The noncommutative plane wave (52) is the kernel of the noncommutative Fourier transform, the transform between the group and algebra representation. We have now all the material to show the form of such noncommutative Fourier transform and to discuss some of its properties. We will discuss also its relation with other known constructions for a Fourier transform on the group, as well as with the Plancherel decomposition into irreducible representations.

\section{A. The NC Fourier transform for $\operatorname{SL}(2, \mathbb{C})$}

In order to write the expression of the noncommutative Fourier transform we first need to evaluate the Haar measure $\mu(g)$ for the Lorentz group in canonical coordinates $k^{i}(g) \equiv\left(\rho^{i}(g), \beta^{i}(g)\right)$, such that $d g=\mu(\vec{\rho}(g), \vec{\beta}(g)) d^{3} \vec{\rho} d^{3} \vec{\beta}$. The calculation is performed in Appendix A 3. From the definition (14), considering the expression of the plane wave (52) and the Haar measure (A19) the Fourier transform $\mathcal{F}: L^{2}(g) \rightarrow L_{\star}^{2}\left(\mathfrak{g}^{*}\right)$ and its inverse $\mathcal{F}^{-1}$ are 


$$
\begin{aligned}
\tilde{\psi}(X) & =\mathcal{F}(\psi)(X)=\int_{G} d^{3} \vec{\rho} d^{3} \vec{\beta} \mathcal{A}^{-1}(\vec{\rho}, \vec{\beta}) e^{i \vec{\rho} \cdot \vec{X}^{J}+i \vec{\beta} \cdot \vec{X}^{N}} \psi(g), \\
\psi(g) & =\mathcal{F}^{-1}(\tilde{\psi})(g) \\
& =\frac{1}{(2 \pi)^{6}} \int_{\mathfrak{g}^{*}} d^{3} X^{J} d^{3} X^{N} \mathcal{A}(\vec{\rho}, \vec{\beta}) e^{-i \vec{p} \cdot \vec{X}^{J}-i \vec{\beta} \cdot \vec{X}^{N}} \star \tilde{\psi}(X),
\end{aligned}
$$

where $(\vec{\rho}, \vec{\beta})=(\vec{\rho}(g), \vec{\beta}(g))$, and the integration measure $d^{3} \vec{\rho} d^{3} \vec{\beta}$ is the standard Lebesgue measure, with the parameters restricted as explained in Sec. III A depending if $G$ is $\operatorname{SL}(2, \mathbb{C})$ or $\operatorname{SO}(3,1): \eta \in[0, \infty)$ and $\phi \in(-2 \pi, 2 \pi]$ for $\operatorname{SL}(2, \mathbb{C})$ while $\phi \in(-\pi, \pi]$ for $\operatorname{SO}(3,1)$, with $\phi^{2}-\eta^{2}=$ $\rho^{2}-\beta^{2}, \phi \eta=\vec{\rho} \cdot \vec{\beta}$. The integration measure $d^{3} X^{J} d^{3} X^{N}$ is the Lebesgue measure $d^{3} X^{J} d^{3} X^{N}$ with $X^{J} \in \mathbb{R}^{3}, X^{N} \in \mathbb{R}^{3}$. As explained in Sec. II, the Fourier transform so defined acts as intertwiner between the group and algebra representation, where the intertwining property can be expressed as $\mathcal{F} \circ \pi_{G}(\hat{T})=\pi_{\mathfrak{g}^{*}}(\hat{T}) \circ \mathcal{F}$, where $\hat{T} \in \mathcal{U}$.

From the definition of the Fourier transform (67), we get the general following properties (we refer to [15] for the general proofs):

(i) The group multiplication from the left is dually represented on $\mathcal{F}(\psi)(X)$ as $\star$-multiplication by $E_{g^{-1}}(X)$ :

$$
\begin{aligned}
\mathcal{F}\left(L_{g} \psi\right)(X) & =\int_{G} d h E_{h}(X) \psi(g h) \\
& =E_{g^{-1}}(X) \star \mathcal{F}(\psi)(X) .
\end{aligned}
$$

(ii) Noticing that $\overline{E_{g}(X)}=E_{g^{-1}}(X)=E_{g}(-X)$ we get the delta function on $L^{2}(g)$

$$
\begin{aligned}
\delta(g) & =\delta^{d}(z(g))=\int_{\mathfrak{g}^{*}} \frac{d^{d} X}{(2 \pi)^{d}} \overline{E_{g}(X)} \\
& =\frac{1}{(2 \pi)^{6}} \int_{\mathfrak{g}^{*}} d^{3} X^{J} d^{3} X^{N} \mathcal{A}(\vec{\rho}, \vec{\beta}) e^{-i \vec{\rho} \cdot \vec{X}^{J}-i \vec{\beta} \cdot \vec{X}^{N}}
\end{aligned}
$$

for coordinates $z(g)$ such that $z(e)=0, \mathcal{L}_{i} z^{j}(e)=\delta_{i}^{j}$, which has the property (see A 5)

$$
\delta(g h)=\mu(z(h))^{-1} \delta^{d}(z(g)+z(h)),
$$

$\mu(z(g))$ being the Haar measure factor $d g=$ $\mu(z(g)) d^{d} z(g)$.

(iii) $\mathcal{F}$ is an isometry from $L^{2}(G)$ to $L_{\star}^{2}\left(\mathfrak{g}^{*}\right)$ in that it preserves the $L^{2}$ norms (6) and (10):

$$
\begin{aligned}
\left\langle\tilde{\psi}, \tilde{\psi}^{\prime}\right\rangle_{\mathfrak{g}^{*}} & :=\int_{\mathfrak{g}^{*}} \frac{d^{d} X}{(2 \pi)^{d}}\left(\overline{\tilde{\psi}(X)} \star \tilde{\psi}^{\prime}(X)\right) \\
& =\int_{G} d \overline{\psi(g)} \psi^{\prime}(g)=\left\langle\psi, \psi^{\prime}\right\rangle_{G},
\end{aligned}
$$

so that we may identify $L_{\star}^{2}\left(\mathfrak{g}^{*}\right)=\mathcal{F}\left(L^{2}(G)\right)$.

(iv) The $\star$-product is dual to the convolution product $*$ on $G$ under the noncommutative Fourier transform:

$$
\begin{aligned}
\tilde{\psi} \star \tilde{\psi}^{\prime} & =\psi \widetilde{* \psi^{\prime}} \\
\text { where } \psi * \psi^{\prime}(g) & =\int_{G} d h \psi\left(g h^{-1}\right) \psi^{\prime}(h) .
\end{aligned}
$$

Moreover one can see that, in canonical coordinates $k^{i}(g) \equiv\left(\rho^{i}(g), \beta^{i}(g)\right)$, the Haar measure coincides with the inverse square of the factor (53) coming from the Duflo map:

$$
\mu(\vec{\rho}(g), \vec{\beta}(g))=\mathcal{A}^{-2}(\vec{\rho}(g), \vec{\beta}(g)) .
$$

This remarkable feature reflects the property (44) of the Duflo map, and from it some interesting consequences arise in the characterization of the Fourier transform:

(i) notice first that the property (73) implies that the inner product of two plane waves respect to $\mathfrak{g}^{*}$, which is the group delta function, becomes the pointwise product between plane waves. Indeed from (69), for $g, h \in G$,

$$
\begin{aligned}
\int_{\mathfrak{g}^{*}} d^{d} X E_{g}(X) \star E_{h}(X) & =\int_{\mathfrak{g}^{*}} d^{d} X E_{g h}(X) \\
& =\delta(g h) .
\end{aligned}
$$

Using the transformation law (70) for canonical coordinates $z(g)=(\vec{\rho}(g), \vec{\beta}(g))$,

$$
\begin{aligned}
\delta(g h) & =\mu^{-1}(z(g)) \delta^{d}(z(g)+z(h)) \\
& =\mu^{-1}(z(g)) \int_{\mathfrak{g}^{*}} d^{d} X e^{i(z(g)+z(h)) \cdot X},
\end{aligned}
$$

where we used the ordinary representation of the delta function $\delta^{d}(z(g)+z(h))$ with respect to the Lebesgue measure $d^{d} X$. Noticing that the last expression is nonzero only for $z(h)=-z(g)$, and that $\mathcal{A}(-z(g))=\overline{\mathcal{A}(z(g))}$, we can rewrite it as

$$
\begin{aligned}
& \mu^{-1}(z(g))\left|\mathcal{A}^{-2}(z(g))\right| \\
& \quad \times \int_{\mathfrak{g}^{*}} d^{d} X \mathcal{A}(z(g)) e^{i z(g) \cdot X} \mathcal{A}(z(h)) e^{i z(h) \cdot X} .
\end{aligned}
$$

But from (73), for the Duflo map, this implies 


$$
\begin{aligned}
\delta(g h) & =\int_{\mathfrak{g}^{*}} d^{6} X E_{g}(X) \star E_{h}(X) \\
& =\int_{\mathfrak{g}^{*}} d^{6} X E_{g}(X) E_{h}(X) .
\end{aligned}
$$

This implies that the Duflo $L_{\star}^{2}$ inner product coincides with the usual $L^{2}$ inner product, and therefore $L_{\star}^{2}\left(\mathfrak{g}^{*}\right) \subseteq L^{2}\left(\mathfrak{g}^{*}\right)$ as an $L^{2}$ norm-complete vector space for the Duflo map. Indeed, considering the representation property (54) it follows by linearity from (76) that for a generic quantization map

$\int_{\mathfrak{g}^{\star}} d^{d} X \tilde{\phi}(X) \star \tilde{\psi}(X)=\int_{\mathfrak{g}^{\star}} d^{d} X(\sigma(i \vec{\partial}) \tilde{\phi}(X)) \tilde{\psi}(X)$,

where $\sigma(z)^{-1}=\mu(z)|\mathcal{A}(z)|^{2}$. Using again (73), for the Duflo map last relation reduces to

$\int_{\mathfrak{g}^{\star}} d^{d} X \tilde{\phi}(X) \star \tilde{\psi}(X)=\int_{\mathfrak{g}^{\star}} d^{d} X \tilde{\phi}(X) \tilde{\psi}(X)$,

showing that under integration, the Duflo $\star$-product coincides with the pointwise product.

(ii) A similar feature characterizes the inner product of two plane waves respect to $G$. It is easy to see from the definition of the Fourier transform that it corresponds to the noncommutative Dirac delta in $L_{\star}^{2}\left(\mathfrak{g}^{*}\right)$

$$
\frac{1}{(2 \pi)^{6}} \int_{\mathrm{SL}(2, \mathbb{C})} d g E_{g}(X) \overline{E_{g}(Y)}=\delta_{\star}(X, Y),
$$

acting as a standard delta distribution with respect to the $\star$-product:

$$
\begin{aligned}
\int_{\mathfrak{g}^{*}} d^{d} X \delta_{\star}(X) \star \tilde{\psi}(X) & =\int_{\mathfrak{g}^{*}} d^{d} X \tilde{\psi}(X) \star \delta_{\star}(X) \\
& =\tilde{\psi}(0),
\end{aligned}
$$

where $\delta_{\star}(X)=\delta_{\star}(X, 0)$. But in particular for the Duflo map it follows from (73) that

$$
\begin{aligned}
& \frac{1}{(2 \pi)^{6}} \int_{\mathrm{SL}(2, \mathbb{C})} d g E_{g}(X) \overline{E_{g}(Y)} \\
& =\frac{1}{(2 \pi)^{6}} \int_{\mathrm{SL}(2, \mathbb{C})} d^{3} \vec{\rho} d^{3} \vec{\beta} e^{i \vec{\rho} \cdot\left(\vec{X}^{J}-\vec{Y}^{J}\right)+i \vec{\beta} \cdot\left(\vec{X}^{N}-\vec{Y}^{N}\right),}
\end{aligned}
$$

i.e., the inner product of two plane waves reduces to the standard orthogonality expression in canonical coordinates with respect to the Lebesgue measure $d^{3} \vec{\rho} d^{3} \vec{\beta}$, and the noncommutative delta function reduces to the standard delta respect to the pointwise product, $\delta_{\star}(X, Y)=\delta(X, Y)$, with the only important caveat that the parameters relative to the compact subgroup have compact range. Specifically, the restriction on the domain of $\phi, \phi \in(-2 \pi, 2 \pi]$ (or $\phi \in(-\pi, \pi]$ for $\mathrm{SO}(3,1)$ ), implies the domain of $(\vec{\rho}, \vec{\beta})$ to be restricted by the condition

$$
\begin{aligned}
& \sqrt{\frac{1}{2}\left(\left(\rho^{2}-\beta^{2}\right)+\sqrt{\left(\rho^{2}-\beta^{2}\right)^{2}+4(\vec{\rho} \cdot \vec{\beta})^{2}}\right)} \\
& \in(-2 \pi, 2 \pi] \quad(\operatorname{or}(-\pi, \pi] \quad \text { for } \operatorname{SO}(3,1)) .
\end{aligned}
$$

Notice that for the special case in which the boost and rotation parameters are collinear, i.e., $\vec{\rho} . \vec{\beta}=\rho \beta$, this expression reduces to a condition on the modulus of the (canonical) rotation parameter

$$
\rho \in[0,2 \pi), \quad(\text { or }[0, \pi) \text { for } \operatorname{SO}(3,1)) .
$$

Thus, (82) tells us that the Duflo noncommutative delta behaves as a standard delta distribution when considering the commutative space of variables $X$, where however due to the restriction on the parameter range associated to the compact subgroup, some of the values of the $X$ spaces are restricted to take discrete values. This is the expected result, due to the compactness of the corresponding sections of the conjugate space. In fact, the same feature was pointed out in previous works concerning noncommutative harmonic analysis on compact groups [18].

\section{B. Relation between the noncommutative Fourier transform and the Fourier expansion in group unitary irreducible representations}

The noncommutative Fourier transform allows to switch between the group representation and the algebra representation of the quantum algebra $\mathfrak{U}$. In standard harmonic analysis, however, functions on the group are expanded in terms of unitary irreducible representations. In that case a different generalization of the Fourier transform is considered, consisting in a unitary map from square-integrable functions $L^{2}(G)$ to square-integrable functions $L^{2}(\hat{G})$ on the Pontryagin dual.

The harmonic analysis on the Lorentz group is developed for instance in [29]. It is shown that a function $\psi(g) \in$ $L^{2}(\operatorname{SL}(2, \mathbb{C}))$ can be expanded in terms of irreducible unitary (infinite dimensional) representations of the principal series, by means of the Plancherel decomposition (and its inverse) 


$$
\begin{aligned}
\hat{\psi}_{j_{1} j_{2} q_{1} q_{2}}^{\chi}= & \int_{\mathrm{SL}(2, \mathbb{C})} d g D_{j_{1} j_{2} q_{1} q_{2}}^{\chi}(g) \psi(g), \\
\psi(g)= & \frac{1}{2} \int_{\mathbb{R}} d r \sum_{m=-\infty}^{+\infty}\left(m^{2}+r^{2}\right) \\
& \times \sum_{j_{1}, j_{2}=|(1 / 2) m|}^{\infty} \sum_{q_{1}=-j_{1}}^{j_{1}} \sum_{q_{1}=-j_{2}}^{j_{2}} \frac{D_{j_{1} j_{2} q_{1} q_{2}}^{\chi}}{}(g) \hat{\psi}_{j_{1} j_{2} q_{1} q_{2}}^{\chi},
\end{aligned}
$$

where $\chi=(m, r)$ labels the representation and, in the principal series, $r$ is a real continuous parameter, while $m$ takes discrete values. $d \chi=d r\left(m^{2}+r^{2}\right)$ is the Plancherel measure, and the Fourier coefficients $\hat{\psi}_{j_{1} j_{2} q_{1} q_{2}}^{\chi}$ are matrix elements of functions $L^{2}(\hat{G}) \cdot D_{j_{1} j_{2} q_{1} q_{2}}^{\chi}(g)$ are matrix elements of irreducible unitary representations in the socalled "canonical basis," spanned by the set $\phi_{q}^{j}(u)=$ $(2 j+1)^{1 / 2} D_{(1 / 2) m, q}^{j}(u), \quad j=\left|\frac{1}{2} m\right|+n, \quad n=0,1,2, \ldots$, $-j \leq q \leq j$, complete in $L_{m}^{2}(u)$, the space of measurable functions $\phi(u)$ on $\mathrm{SU}(2)$ covariant on the right cosets of $\mathrm{U}(1)$ as $\phi(\gamma u)=e^{i m \omega} \phi(u)$, with $u \in \mathrm{SU}(2)$ and $\gamma=$ $\left(\begin{array}{c}\exp (i \omega) \\ 0\end{array} \stackrel{0}{\exp (-i \omega)}\right)$. Here $D_{q_{1} q}^{j_{1}}\left(u_{1}\right)$ define the SU(2) unitary irreducible representations (Wigner matrices), and the matrices $D_{j_{1} j_{2} q_{1} q_{2}}^{\chi}(g)$ can be decomposed as

$$
D_{j_{1} j_{2} q_{1} q_{2}}^{\chi}(g)=\sum_{q} D_{q_{1} q}^{j_{1}}\left(u_{1}\right) D_{q q_{2}}^{j_{2}}\left(u_{2}\right) d_{j_{1} j_{2} q}^{\chi}(\eta)
$$

where $u_{1}, u_{2} \in \mathrm{SU}(2)$ and $\eta$ is the "boost" parameter, and $d_{j_{1} j_{2} q}^{\chi}(\eta)$ can be written in the integral representation

$$
\begin{aligned}
d_{j_{1} j_{2} q}^{\chi}(\eta)= & \left(2 j_{1}+1\right)^{1 / 2}\left(2 j_{2}+1\right)^{1 / 2} \int_{0}^{1} d t d_{(1 / 2) m, q}^{j_{1}}(2 t-1) \\
& \times d_{(1 / 2) m, q}^{j_{2}}\left(2 t_{d}-1\right)\left[t e^{-\eta}+(1-t) e^{\eta}\right]^{(i / 2) r-1} .
\end{aligned}
$$

Thus, the above makes use of the fact that the space $L_{m}^{2}(u)$ decomposes into a direct orthogonal sum of $(2 j+1)$-dimensional Hilbert spaces $\mathcal{H}_{j}$ carrying each an irreducible representation of $\mathrm{SU}(2): L_{m}^{2}(u)=\bigoplus_{j=\left|\frac{1}{2} m\right|}^{\infty} \mathcal{H}_{j}$.

The Fourier transform (85) realizes the decomposition of the regular (right and left) representation, carried by the Hilbert space $L^{2}(\operatorname{SL}(2, \mathbb{C}))$, into irreducible unitary representations of the principal series. More precisely, consider the kernel $K_{\psi}\left(u_{1}, u_{2} \mid \chi\right)$ of the Fourier transform (85) defined by [here $d \mu(u)$ is the Haar measure for SU(2)]

$$
\hat{\psi}_{j_{1} j_{2} q_{1} q_{2}}^{\chi}=\int d \mu\left(u_{1}\right) d \mu\left(u_{2}\right) \overline{\phi_{q_{1}}^{j_{1}}\left(u_{1}\right)} K_{\psi}\left(u_{1}, u_{2} \mid \chi\right) \phi_{q_{2}}^{j_{2}}\left(u_{2}\right),
$$

and denote, for any representation $\chi$ and $-\chi$ of the principal series, $L_{m}^{2}\left(U_{1}\right)_{q}^{j}$ and $L_{m}^{2}\left(U_{2}\right)_{q}^{j}$ the Hilbert spaces of, respectively, the measurable functions ${ }^{12}$

$$
\begin{aligned}
\phi_{\psi}\left(u_{1} \mid \chi\right)_{q}^{j} & =\int d \mu\left(u_{2}\right) K_{\psi}\left(u_{1}, u_{2} \mid \chi\right) \phi_{q}^{j}\left(u_{2}\right), \\
\phi_{\psi}\left(u_{2} \mid \chi\right)_{q}^{j} & =\int d \mu\left(u_{1}\right) \overline{\phi_{q}^{j}\left(u_{2}\right)} K_{\psi}\left(u_{1}, u_{2} \mid-\chi\right) .
\end{aligned}
$$

One can show that the image of $L^{2}(\operatorname{SL}(2, \mathbb{C}))$ by the Fourier transform (85) can be mapped isometrically into a Hilbert space $\mathcal{H}=\int_{r \geq 0}^{\oplus} d \chi \bigoplus_{j=\frac{1}{2} m \mid}^{\infty} \bigoplus_{q=-j}^{j} L_{m}^{2}\left(U_{1}\right)_{q}^{j} \quad$ (or $\mathcal{H}=\int_{r \geq 0}^{\oplus} d \chi \bigoplus_{j=\left|\frac{1}{2} m\right|}^{\infty} \bigoplus_{q=-j}^{j} L_{m}^{2}\left(U_{2}\right)_{q}^{j}$ ) such that a left (or right) translation in $L^{2}(\operatorname{SL}(2, \mathbb{C}))$ generates irreducible unitary representations $\chi$ of the principal series in $L_{m}^{2}\left(U_{1}\right)_{q}^{j}\left(L_{m}^{2}\left(U_{2}\right)_{q}^{j}\right.$ respectively $)$

By means of Eqs. (67) and (85) one can obtain the relation between the expansion of a function $L^{2}(\operatorname{SL}(2, \mathbb{C}))$ in terms of noncommutative plane waves and the one in terms of irreducible group representations (Plancherel modes):

$$
\begin{aligned}
& \hat{\psi}_{j_{1} j_{2} q_{1} q_{2}}^{\chi}=\frac{1}{(2 \pi)^{6}} \int_{\mathfrak{g}^{*}} d^{3} X^{J} d^{3} X^{N} D_{j_{1} j_{2} q_{1} q_{2}}^{\chi}(X) \star \tilde{\psi}(X), \\
& \tilde{\psi}(X)=\frac{1}{2} \int_{\mathbb{R}} d r \sum_{m=-\infty}^{+\infty}\left(m^{2}+r^{2}\right) \\
& \times \sum_{j_{1}, j_{2}=|(1 / 2) m|}^{\infty} \sum_{q_{1}=-j_{1}}^{j_{1}} \sum_{q_{1}=-j_{2}}^{j_{2}} \overline{D_{j_{1} j_{2} q_{1} q_{2}}^{\chi}(X)} \hat{\psi}_{j_{1} j_{2} q_{1} q_{2}}^{\chi} \\
& D_{j_{1} j_{2} q_{1} q_{2}}^{\chi}(X)=\int_{\mathrm{SL}(2, \mathbb{C})} d g D_{j_{1} j_{2} q_{1} q_{2}}^{\chi}(g) \overline{E_{g}(X)} .
\end{aligned}
$$$$
\text { C. Comparison with existing results on }
$$$$
\text { noncommutative Fourier transform }
$$
for noncompact groups

As we discussed in the Introduction, the noncommutative Fourier transform has played an important role in the context of quantum gravity, and more specifically in effective models based on the idea of spacetime noncommutativity. In particular, when the noncommutativity of spacetime coordinate operators is of Lie algebra type, the associated momentum space can be described as a curved manifold with a Lie group structure. The idea of a curved momentum space dates back to M. Born [36], as a way to make more symmetric the role of configuration space (generically

\footnotetext{
${ }^{12}$ The Hilbert spaces $L_{m}^{2}\left(U_{1}\right)_{q}^{j}$ and $L_{m}^{2}\left(U_{2}\right)_{q}^{j}$ are equivalent, as it exists an intertwining operator between the $\chi$ and $-\chi$ representations.
} 
curved, in a GR setting) and momentum space, as a possible key to quantum gravity. The same idea was later formalized more rigorously in the language of Hopf-algebras (quantum groups) [37], through the introduction of a suitable notion of integration. It has been then further investigated and developed for several examples of noncommutative spaces (and spacetimes).

Examples of noncommutativity with a noncompact group manifold for the momenta have been also considered ${ }^{13}$. Particularly relevant for our analysis is a work investigating a Snyder [38] kind of noncommutativity in three dimensions [39]. From a group theoretical point of view, it is possible indeed to consider Snyder noncommutative spacetime to be described by the quotient $\mathfrak{g} \mathfrak{o}(4,1) / \mathfrak{g} \mathfrak{o}(3,1)$, so that the associated momentum space consists in the homogeneous space $\mathrm{SO}(4,1) / \mathrm{SO}(3,1)$, i.e., de Sitter space. In [39] a "Euclidean" version of threedimensional Snyder spacetime is considered, where momentum space consists in the homogeneous space given by the coset $\mathrm{SO}(3,1) / \mathrm{SO}(3)$, the three-dimensional hyperboloid $\mathcal{H}_{3}$. The case treated fits well with the context of the work we have presented here, since the $\star$-product introduced in Snyder configuration space and the noncommutative Fourier transform relating it to the curved momentum space are induced by the group and algebra structure of the Lorentz group. They have not been directly derived from a choice of quantization map, but rather postulated at the onset of the analysis. Using our framework, however, this quantization map can be in principle read out of the postulated $\star$-product. In particular, the authors of [39] consider two different $\star$-products (a nonassociative and an associative one), corresponding to two different parametrizations of the $\mathrm{SO}(3)$ and $\mathrm{SO}(3,1) / \mathrm{SO}(3)$ sectors, followed by the application of the symmetric map (42) to the two sectors separately, i.e., to a ordering prescription for the noncommutative operators corresponding to the Cartan decomposition. It is not clear what are the properties of the chosen quantization maps nor why they would be preferable to the Duflo map, from a mathematical perspective. The more detailed relation with our construction will be studied in future work. We will discuss in the next section the implementation of our noncommutative Fourier transform, based on the Duflo quantization map, for a Cartan decomposition of $\mathrm{SO}(3,1)$, and for the homogeneous space $\mathcal{H}_{3} \sim \mathrm{SO}(3,1) / \mathrm{SO}(3)$. This may facilitate a future detailed comparison of the construction presented in this paper and the one of [39].

Another much studied example of spacetime noncommutativity associated to a curved noncompact momentum space related to the Lorentz group, is that of $\kappa$-Minkowski [40-42] (with its associated Hopf-algebra of symmetries, $\kappa$-Poincaré). In this case, the noncommutativity is of Lie

\footnotetext{
${ }^{13}$ We mention also the work [48], where the non-commutative Fourier transform for the $\operatorname{SL}(2, \mathfrak{R})$ group is discussed, in connection with some known results for $(2+1) D$ gravity.
}

algebra type, and the associated momentum space is the group manifold $A N_{3}$, corresponding to half of de Sitter space [43]. The properties of the noncommutative Fourier transform for $\kappa$-Minkowski have been considered in several studies. The Hopf-algebra point of view, where a "time ordering" prescription is used to define the noncommutative plane wave is taken in [7-9], and the corresponding quantization map discussed in [44]. The point of view of the group structure of the momentum manifold is instead central in [6], and in [45] its formulation in terms of group field theory has been analyzed. In our language we can understand the noncommutative Fourier transform discussed in these works, which is the one associated to the so-called "bicrossproduct" basis of $\kappa$-Poincaré [42], to correspond to a quantization map consisting in a specific time-ordering prescription for the noncommutative operators (see again [44], where alternative choices of orderings are also considered). Once more, while the physical idea behind the time-ordering is transparent, it is not clear if this leads to any advantage from the mathematical perspective of the algebra of quantum observables, compared to the Duflo map.

\section{Aside the noncommutative Fourier transform for the homogeneous space $\operatorname{SL}(2, \mathbb{C}) / \mathrm{SU}(2)$}

Using the results obtained for the Lorentz group, we can describe as well the properties of the noncommutative Fourier transform for its homogeneous spaces. We discuss here only the homogeneous space $\mathcal{H}_{3} \cong \mathrm{SL}(2, \mathbb{C}) / \mathrm{SU}(2)$ (or $\mathrm{SO}(3,1) / \mathrm{SO}(3)$ ). This is relevant for the physical applications mentioned in the previous subsections, and one more physical application of our formalism using the same homogeneous space will be given in the next section. In fact, it is also an interesting domain for spin foam models and group field theory constructions for Lorentzian quantum gravity in $4 \mathrm{~d}[11,46]$.

Consider the decomposition of an element of $\operatorname{SL}(2, \mathbb{C})$

$$
g=k h=\exp (i \overrightarrow{\mathfrak{b}} \cdot \vec{N}) \exp (i \overrightarrow{\mathfrak{r}} \cdot \vec{J})
$$

where $h=\exp (i \overrightarrow{\mathfrak{r}} \cdot \vec{J}) \in \mathrm{SU}(2)$, corresponding to the Cartan decomposition. Parametrizing a generic point on $\mathcal{H}_{3}$ as

$$
q=q_{0} \mathbb{1}-\vec{q} \cdot \vec{\sigma} \equiv\left(q_{0}, \vec{q}\right),
$$

$q$ is defined by the $\operatorname{SL}(2, \mathbb{C})$ action on the origin $q_{a}=(1, \overrightarrow{0}) \equiv \mathbb{1}$ :

$$
\begin{aligned}
q & =g q_{a} g^{\dagger}=k q_{a} k^{\dagger}=\cosh (\mathfrak{b}) \mathbb{1}-\sinh (\mathfrak{b}) \hat{\mathfrak{b}} \cdot \vec{\sigma} \\
& \equiv(\cosh (\mathfrak{b}), \sinh (\mathfrak{b}) \hat{\mathfrak{b}}) .
\end{aligned}
$$


Thus, the quotient space $\mathrm{SL}(2, \mathbb{C}) / \mathrm{SU}(2)$ can be identified with the $\operatorname{coset}^{14} g H, H \equiv \mathrm{SU}(2)$. Accordingly, in the splitting (92) the boost element $k$ is a representative of the set of equivalence classes $[k]:=\{k h, \forall h \in \mathrm{SU}(2)\}$ defining $g H$.

So, we can identify functions $f_{\mathcal{H}_{3}}(q)$ on $\mathcal{H}_{3}$ with functions $f(g H)$ on the coset $\mathrm{SL}(2, \mathbb{C}) / \mathrm{SU}(2)$, constant on the orbits of $\mathrm{SU}(2)$, through the projection

$$
\begin{gathered}
f_{\mathcal{H}_{3}}(q) \equiv f(g H)=\int_{\mathrm{SU}(2)} \operatorname{dhf}(g h), \quad q \in \mathcal{H}_{3}, \\
g \in \mathrm{SL}(2, \mathbb{C}), \quad h \in H \equiv \mathrm{SU}(2)
\end{gathered}
$$

where, for integrable functions on $\mathcal{H}_{3}$, the Haar measure $d g$ induces a $\operatorname{SL}(2, \mathbb{C})$-invariant Haar measure $d q$ on $\mathcal{H}_{3}$ such that [47]

$$
\int_{\mathrm{SL}(2, \mathbb{C})} d g f(g)=\int_{\mathcal{H}_{3}} d q \int_{\mathrm{SU}(2)} d h f(g h) .
$$

The Haar measure for the decomposition (92) factorizes (see A 4) into the product of the measure on $\mathcal{H}_{3}$ and the measure on the subgroup $\mathrm{SU}(2)$

$d g=d q d h, \quad q \in \mathcal{H}_{3}, \quad h \in \mathrm{SU}(2), \quad$ with

$d q=\frac{\sinh ^{2}(\mathfrak{b})}{\mathfrak{b}^{2}} d^{3} \overrightarrow{\mathfrak{b}}, \quad d h=4 \frac{\sin ^{2}\left(\frac{1}{2} \mathfrak{r}\right)}{\mathfrak{r}^{2}} d^{3} \overrightarrow{\mathfrak{r}}$.

The plane wave can be written in Cartan coordinates as

$$
E_{k h}(X)=\mathcal{A}(\vec{\rho}(\overrightarrow{\mathfrak{b}}, \overrightarrow{\mathfrak{r}}), \vec{\beta}(\overrightarrow{\mathfrak{b}}, \overrightarrow{\mathfrak{r}})) e^{i \vec{\rho}(\overrightarrow{\mathfrak{b}}, \overrightarrow{\mathfrak{r}}) \cdot \vec{X}^{J}+i \vec{\beta}(\overrightarrow{\mathfrak{b}}, \overrightarrow{\mathfrak{r}}) \cdot \vec{X}^{N}}
$$

where $(\vec{\rho}(\mathfrak{b}, \mathfrak{x}), \vec{\beta}(\mathfrak{b}, \mathfrak{r}))$ are given by the $\mathrm{BCH}$ formula (A20). Notice now that from (A20) it follows that

for $\mathfrak{b}=0, \quad \vec{\rho}=\overrightarrow{\mathfrak{r}}, \quad \phi=\mathfrak{r}, \quad \beta=\eta=0$,

for $\mathfrak{r}=0, \quad \vec{\beta}=\overrightarrow{\mathfrak{b}}, \quad \eta=\mathfrak{b}, \quad \phi=\rho=0$,

so that, from the expression of the Duflo factor (53),

$$
\begin{aligned}
& \mathcal{A}(\vec{\rho}(\mathfrak{b}=0, \overrightarrow{\mathfrak{r}}), \quad \vec{\beta}(\mathfrak{b}=0, \overrightarrow{\mathfrak{r}}))=\mathcal{A}(\overrightarrow{\mathfrak{r}}, 0)=\frac{\mathfrak{r}^{2}}{4 \sin ^{2}\left(\frac{1}{2} \mathfrak{r}\right)} \\
& \mathcal{A}(\vec{\rho}(\overrightarrow{\mathfrak{b}}, \mathfrak{r}=0), \quad \vec{\beta}(\overrightarrow{\mathfrak{b}}, \mathfrak{r}=0))=\mathcal{A}(0, \overrightarrow{\mathfrak{b}})=\frac{\mathfrak{b}^{2}}{4 \sinh ^{2}\left(\frac{1}{2} \mathfrak{b}\right)}
\end{aligned}
$$

Considering that if $h=\mathbb{1} \overrightarrow{\mathfrak{r}}=0$ and if $k=\mathbb{1} \overrightarrow{\mathfrak{b}}=0$, we can write the plane wave for the particular group elements $\left.k h\right|_{h=\mathbb{1}}$ and $\left.k h\right|_{k=\mathbb{1}}$ as

\footnotetext{
${ }^{14}$ The decomposition works similarly for $\mathcal{H}_{3} \cong \mathrm{SO}(3,1) / \mathrm{SO}(3)$, the difference being again in the range of the rotation parameter. In this case (see also Sec. III A) a point in the space $\mathcal{H}_{3}$ is defined by the 4 -vector $\left(q_{0}, \vec{q}\right)$, while the action on the origin $q_{0}=(1, \overrightarrow{0})$ is given by $q=g q_{a}$ for $g \in \mathrm{SO}(3,1)$. We omit in the following the distinction between the two cases unless needed.
}

$$
\begin{aligned}
& E_{k}(X)=\frac{\mathfrak{b}^{2}}{4 \sinh ^{2}\left(\frac{1}{2} \mathfrak{b}\right)} e^{i \overrightarrow{\mathfrak{b}} \cdot \vec{X}^{N}} \\
& E_{h}(X)=\frac{\mathfrak{r}^{2}}{4 \sin ^{2}\left(\frac{1}{2} \mathfrak{r}\right)} e^{i \overrightarrow{\mathfrak{r}} \cdot \vec{X}^{J}}
\end{aligned}
$$

and, from the property (21) of $\star$-product,

$$
E_{k h}(X)=E_{k}(X) \star E_{h}(X) .
$$

We can now use Eq. (95) and the expression for the (inverse) Fourier transform (67) to write

$$
\begin{aligned}
f_{\mathcal{H}_{3}}(q) & \equiv \int_{\mathrm{SU}(2)} d h f(g h) \\
& =\frac{1}{(2 \pi)^{6}} \int_{\mathrm{SU}(2)} d h \int_{\mathfrak{g}^{*}} d^{d} X \overline{E_{g h}(X)} \star \tilde{f}(X),
\end{aligned}
$$

which can be rewritten, using (notice that $\overline{E_{g h}(X)}=$ $\left.E_{h^{-1} g^{-1}}(X)=\overline{E_{h}(X)} \star \overline{E_{g}(X)}\right)$, as

$$
f_{\mathcal{H}_{3}}(q) \equiv \frac{1}{(2 \pi)^{6}} \int_{\mathfrak{g}^{*}} d^{d} X \int_{\mathrm{SU}(2)} d h \overline{E_{h}(X)} \star \overline{E_{g}(X)} \star \tilde{f}(X) .
$$

Using (101) and (97) we notice that the first term reduces to the ordinary delta function (with compact range) on the "rotation" sector of the algebra

$$
\frac{1}{(2 \pi)^{3}} \int_{\mathrm{SU}(2)} d h \overline{E_{h}(X)}=\frac{1}{(2 \pi)^{3}} \int_{\mathrm{SU}(2)} d^{3} \overrightarrow{\mathfrak{r}} e^{-i \overrightarrow{\mathfrak{r}} \cdot \vec{X}^{J}}=\delta^{3}\left(\vec{X}^{J}\right)
$$

with $|\vec{r}| \leq 2 \pi$ [or $|\vec{r}| \leq \pi$ for $\mathrm{SO}(3,1)]$. We can use now the property of the Duflo $\star$-product under integral (79) to eliminate the first $\star$-product in (104) to obtain

$$
f_{\mathcal{H}_{3}}(q) \equiv \frac{1}{(2 \pi)^{3}} \int_{\mathfrak{g}^{*}} d^{d} X \delta^{3}\left(\vec{X}^{J}\right) \overline{E_{g}(X)} \star \tilde{f}(X) .
$$

Notice that in this formula, as in the previous ones leading to it, the $\star$-product used remains the one of $\operatorname{SL}(2, \mathbb{C})$, and all functions are treated as functions on the $T^{*}(\operatorname{SL}(2, \mathbb{C}))$ phase space, only appropriately restricted in their dependence on the domain. ${ }^{15}$

\footnotetext{
${ }^{15}$ Notice also the slight abuse of notation: the pointwise product is between the $\star$-function defined by (105) (corresponding, as a function on $\mathbb{R}^{3}$, to the usual delta function), and the function obtained by $\star$-multiplying the noncommutative plane wave with the function $\tilde{f}$; this pointwise product is then evaluated on the point $X$.
} 


\section{AN EXAMPLE: PARTICLE ON THE HYPERBOLOID}

We will consider now the motion of a free particle on a three-dimensional (spatial) hyperboloid, as a very simple example of application of our formalism in a well-understood context. The quantization of the system can be described in terms of a path-integral formulation. We will show that, by means of the noncommutative Fourier transform, the quantum propagator can be easily formulated in the algebra representation, as its expression assumes, in this simple case, the form one would expect from the classical action by identifying the (noncommutative) classical momenta with the algebra elements. In this sense, the main virtue of the noncommutative Fourier transform and of the algebra representation is to allow for a description of the quantum system in which the underlying classical theory is manifest.

The path-integral formulation of quantum mechanics in terms of noncommutative momenta was described in [49] for the case of $\mathrm{SO}(3)$, and the corresponding propagator for a free particle on a sphere was derived. In dealing with the homogeneous space $\mathcal{H}_{3} \cong \mathrm{SL}(2, \mathbb{C}) / \mathrm{SU}(2)$ [or $\mathrm{SO}(3,1) / \mathrm{SO}(3)$ ], we will refer to [49] for the details on the general characterization of quantum mechanics in noncommutative momentum basis.

A remark on notation: we characterize the spatial hyperboloid introducing a length scale $\ell$, corresponding to the radius of curvature, as $y=\ell q=\left(\ell q_{0}, \ell \vec{q}\right)$, so that the (unit) hyperboloid defined by (94) parametrizes the surface $y_{\mu} y^{\mu}=y_{0}^{2}-\vec{y}^{2}=\ell^{2}$. Similarly we define the dimensional coordinates on $\mathcal{H}_{3} \vec{x}=\ell \overrightarrow{\mathfrak{b}}$.

\section{A. The propagator in the group representation}

Considering the characterization of the homogeneous space $\mathcal{H}_{3} \cong \mathrm{SL}(2, \mathbb{C}) / \mathrm{SU}(2)$ of Sec. IV D, the finite time propagator is defined as the expectation value in the Hilbert space $L^{2}\left(\mathcal{H}_{3}\right)$

$$
K\left(q^{\prime \prime}, q^{\prime} ; t^{\prime \prime}-t^{\prime}\right)=\left\langle q^{\prime \prime}\left|U\left(t^{\prime \prime}-t^{\prime}\right)\right| q^{\prime}\right\rangle .
$$

where $|q\rangle$ are vectors in $L^{2}\left(\mathcal{H}_{3}\right)$, and the time evolution operator is defined in terms of the particle momentum $\boldsymbol{p}$ as

$$
U(t)=\exp \left(-i \frac{p^{2} t}{2 m \hbar}\right)
$$

The propagator can be described in the group representation by noticing $[50,51]$ that the finite time propagator (107) can be expressed as the convolution in the group manifold

$$
\begin{aligned}
K\left(q^{\prime \prime}, q^{\prime} ; t\right)= & \lim _{N \rightarrow \infty} \int d g_{1} \cdots \int d g_{N-1} K_{\epsilon}\left(g_{0}^{-1} g_{1}\right) \cdots \\
& \times K_{\epsilon}\left(g_{N-1}^{-1} g_{N}\right), \\
& \left(q_{j}=g_{j} q_{a} g_{j}^{\dagger}, q_{N}=q^{\prime \prime}, q_{0}=q^{\prime}\right)
\end{aligned}
$$

of a spherical function $K_{\epsilon}(g)$ (the short time propagator), satisfying

$$
K_{\epsilon}(g)=K_{\epsilon}\left(h_{1}^{-1} g h_{2}\right), \quad \forall h_{1}, h_{2} \in \mathrm{SU}(2) .
$$

This is our object of interest. We will give its explicit expression in the following, as well as its decomposition in irreducible representation of the group, the analogue of the Peter-Weyl decomposition for compact groups. This will have to be compared to the one obtained via noncommutative Fourier transform. In order to do so, we have to introduce some more harmonic analysis on the hyperboloid.

Functions on the group, satisfying the relation (110), i.e., constant in the two-sided coset $H g H$ [for $H=\mathrm{SU}(2)$ ], can be expanded in terms of zonal spherical functions $\mathcal{D}_{00}^{l}(g)$ [50-52]. Spherical representations (or class 1 representations) $\mathcal{D}^{l}$ of $G$ are unitary irreducible representations which have null vectors $\left|\psi_{\alpha}\right\rangle$ invariant under transformations of a subgroup of $G$. $\mathrm{SU}(2)$ is a massive subgroup of $\operatorname{SL}(2, \mathbb{C})$, meaning that there is only one vector $\left|\psi_{0}\right\rangle$ in any $\mathcal{D}^{l}$ invariant under $\mathrm{SU}(2)$. Then one can define spherical functions

$$
\psi^{l}(g)=\left\langle\psi\left|\mathcal{D}^{l}(g)\right| \psi_{0}\right\rangle
$$

such that $\psi^{l}(g h)=\psi^{l}(g)$, i.e., constant in the left coset $g H$, so that $\psi^{l}(g)$ can be considered as functions on the homogeneous space $\mathcal{H}_{3}$. Choosing a basis $\left\{\left|u_{i}\right\rangle\right\}$ such that $\left|u_{0}\right\rangle=\left|\psi_{0}\right\rangle$, the matrix elements of $\mathcal{D}^{l}(g)$ given by

$$
\mathcal{D}_{m 0}^{l}(g)=\left\langle u_{m}\left|\mathcal{D}^{l}(g)\right| u_{0}\right\rangle
$$

are called associated spherical functions. A zonal spherical function $\mathcal{D}_{00}^{l}(g)$ is thus an associated spherical function constant on the two-sided coset $\mathrm{HgH}$, i.e., such that for any $h, h^{\prime} \in \mathrm{SU}(2) \mathcal{D}_{00}^{l}\left(h g h^{\prime}\right)=\mathcal{D}_{00}^{l}(g)$.

For the spherical principal series the parameter $l$ labeling the representation is continuous and takes the value $l=$ $-1+i r(r \geq 0)$. In terms of zonal spherical functions the Fourier expansion of a function $f(g)$ invariant on the twosided coset $\mathrm{HgH}$ takes the simpler expression

$$
\begin{aligned}
f(g) & =\frac{1}{(2 \pi)^{2}} \int_{0}^{\infty} d r d_{l} \hat{f}^{l} \mathcal{D}_{00}^{l}(g), \\
\hat{f}^{l} & =\int d g f(g) \mathcal{D}_{00}^{l}\left(g^{-1}\right),
\end{aligned}
$$


where $\overline{\mathcal{D}_{00}^{l}}(g)=\mathcal{D}_{00}^{l}\left(g^{-1}\right)$, and $d_{l}$ is a "dimension" factor defined by the relation

$$
\int_{\mathrm{SL}(2, \mathbb{C})} \mathcal{D}_{00}^{l}(g) \mathcal{D}_{00}^{l^{\prime}}\left(g^{-1}\right)=\frac{1}{d_{l}} \delta\left(l-l^{\prime}\right) .
$$

The spherical functions are eigenfunctions of the Laplace-Beltrami operator on $\mathcal{H}_{3}$, that can be identified with the operator $\boldsymbol{p}^{2}$ in the time evolution (108), and corresponds to a Casimir of the Lorentz algebra, restricted to its boost components.

The zonal spherical function coincides with the matrix element $d_{000}^{(0,2 r)}(\eta)$ of the $\operatorname{SL}(2, \mathbb{C})$ representations defined in (87) and takes the explicit form

$$
\mathcal{D}_{00}^{l}(\mathfrak{b}(g))=d_{000}^{(0,2 r)}(\eta(g))=\frac{\sin (r \eta)}{r \sinh (\eta)} .
$$

The dimension factor can be evaluated to $d_{l}=r^{2}$.

In the group representation (i.e., configuration space), and using polar coordinates, the short time propagator takes the form $[50,51]$

$$
\begin{aligned}
K\left(q, q^{\prime} ; \epsilon\right) & =\left\langle q^{\prime \prime}|U(\epsilon)| q^{\prime}\right\rangle \\
& =\left(\frac{m \ell}{2 \pi i \hbar \epsilon}\right)^{\frac{3}{2}} \exp \left(\frac{i}{\hbar}\left(\frac{m \ell^{2}}{\epsilon}(\cosh \Theta-1)+\frac{\hbar^{2} \epsilon}{8 m \ell^{2}}\right)\right),
\end{aligned}
$$

where $\Theta$ is the angle of the hyperbolic rotation transforming $q^{\prime}$ into $q: q=g q^{\prime}$. As shown in Appendix $\mathrm{C} 1$ the angle $\Theta$ coincides with the modulus of the hyperbolic rotation $\mathfrak{b}$ in (94) (and then with $\eta$, when restricting to the hyperboloid).

The expansion of the propagator in terms of zonal spherical representations (the analogue of the Peter-Weyl decomposition for compact Lie groups) takes instead the expression $[50,51]$

$$
\begin{aligned}
K\left(q^{\prime \prime}, q^{\prime} ; t\right)= & \frac{1}{2 \pi^{2}} \int_{0}^{\infty} d r \exp \left(-i \frac{\hbar r^{2}}{2 m \ell^{2}} t\right) \\
& \times r \frac{\sin \left(x\left(g_{0}^{-1} g_{N}\right) r / \ell\right)}{\sinh \left(x\left(g_{0}^{-1} g_{N}\right) / \ell\right)},
\end{aligned}
$$

with $x=|\vec{x}|$.

This is the expression we would have to compare with the one obtained via noncommutative Fourier transform.

\section{B. The propagator in the noncommutative momentum representation}

We identify the particle momenta in the algebra representation with the operators $\hat{X}^{N} \in \mathcal{Q}\left(\mathcal{A}_{\mathfrak{g}^{*}}\right)$ relative to the "boost" sector, through the rescaling

$$
\hat{P}=\frac{\hbar}{\ell} \hat{X}^{N}
$$

while we denote $\hat{R}=\frac{\hbar}{\ell} \hat{X}^{J}$ the "momenta" relative to the rotation sector (which are trivial when we restrict to the hyperboloid).

Following the construction of [49], we define a set of states $\left\{|\vec{P}, \vec{R}\rangle \mid \vec{P}, \vec{R} \in \mathbb{R}_{\star}^{6}\right\}$ in the noncommutative momentum basis, by their inner product with the group basis

$$
\langle g \mid \vec{P}, \vec{R}\rangle \equiv E_{g}(\vec{P}, \vec{R})=\exp _{\star}\left(i \frac{\ell}{\hbar}(\vec{\beta} \cdot \vec{P}+\vec{\rho} \cdot \vec{R})\right)
$$

By virtue of properties (69) and (80) they form a complete and orthonormal basis respect to the $\star$-product:

$$
\begin{aligned}
& \left\langle\vec{P}, \vec{R} \mid \vec{P}^{\prime}, \vec{R}^{\prime}\right\rangle=\left(\frac{2 \pi \hbar}{\ell}\right)^{6} \delta_{\star}\left(\vec{P}-\vec{P}^{\prime}, \vec{R}-\vec{R}^{\prime}\right), \\
& \int_{\mathbb{R}_{\star}^{6}} \frac{d^{3} \vec{P} d^{3} \vec{R}}{(2 \pi \hbar / \ell)^{6}}|\vec{P}, \vec{R}\rangle \star\langle\vec{P}, \vec{R}|=\hat{\mathbb{1}} .
\end{aligned}
$$

On this Hilbert space, corresponding to the algebra representation of the system, we can define the time evolution operator on the Lorentz group (108) to be

$$
U(t)=-\frac{i t}{2 m \hbar}\left(\hat{P}^{2}-\hat{R}^{2}\right)
$$

where $\hat{\mathcal{C}}=\hat{P}^{2}-\hat{R}^{2}$ is the quadratic Casimir of the quantum Lorentz algebra. The full propagator is

$$
\begin{aligned}
K_{G}\left(g^{\prime \prime}, g^{\prime} ; t\right) & =\left\langle g^{\prime \prime}|U(t)| g^{\prime}\right\rangle \\
& =\int_{\mathbb{R}_{\star}^{6}} \frac{d^{3} \vec{P} d^{3} \vec{R}}{(2 \pi \hbar / \ell)^{6}}\left\langle g^{\prime \prime} \mid P, R\right\rangle \star\left\langle P, R|U(t)| g^{\prime}\right\rangle \\
& =\int_{\mathbb{R}_{\star}^{6}} \frac{d^{3} \vec{P} d^{3} \vec{R}}{(2 \pi \hbar / \ell)^{6}} E_{g^{\prime \prime}}(\vec{P}, \vec{R}) \star e_{\star}^{-\frac{i}{2 m \hbar} \sum_{i}\left(P_{i} \star P_{i}-R_{i} \star R_{i}\right)} \\
& \star E_{g^{\prime}}(\vec{P}, \vec{R})
\end{aligned}
$$

The expression $\sum_{i}\left(P_{i} \star P_{i}-R_{i} \star R_{i}\right)$ is the quadratic Casimir in the algebra representation $\mathcal{C}_{\star}$, obtained with the inverse Duflo map, and reduces to [see (B31)]

$$
\mathcal{C}_{\star}=\mathcal{D}^{-1}(\hat{\mathcal{C}})=\sum_{i}\left(P_{i} \star P_{i}-R_{i} \star R_{i}\right)=\vec{P}^{2}-\vec{R}^{2}+\frac{\hbar^{2}}{\ell^{2}}
$$

Notice that $\mathcal{C}_{\star} \star$-commutes with functions of $X$ and we can rewrite the propagator as 


$$
\begin{aligned}
K_{G}\left(g^{\prime \prime}, g^{\prime} ; t\right) & =\int_{\mathbb{R}_{\star}^{6}} \frac{d^{3} \vec{P} d^{3} \vec{R}}{(2 \pi \hbar / \ell)^{6}} e_{\star}^{-\frac{i}{2 m \hbar} \mathcal{C}_{\star}} \star E_{g^{\prime \prime} g^{\prime-1}}(P, R) \\
& =K_{G}\left(g=g^{\prime \prime} g^{\prime-1} ; t\right)
\end{aligned}
$$

where we used that $\overline{E_{g}(X)}=E_{g^{-1}}(X)$.

Now we exploit relation (96) to project the propagator on the homogeneous space $\mathcal{H}_{3}$ (see also Appendix $\mathrm{C} 2$ and [53]):

$$
K\left(q^{\prime \prime}, q^{\prime} ; t\right)=\int_{\mathrm{SU}(2)} d h K_{G}\left(g=g^{\prime \prime} g^{\prime-1} ; t\right)
$$

where $q^{\prime \prime}=g^{\prime \prime} q_{a} g^{\prime \prime \dagger}$ and $q^{\prime}=g^{\prime} q_{a} g^{\prime \dagger}$. Following the discussion of Sec. IV D, we rewrite the plane wave exploiting the splitting (92) and notice that the "boost" part of the plane wave now takes the form (100), in physical coordinates $\vec{x}(g)=\ell \overrightarrow{\mathfrak{b}}(g)$,

$$
E_{k}(\vec{P}, \vec{R})=\frac{(x / 2 \ell)^{2}}{\sinh ^{2}(x / 2 \ell)} e^{i \vec{x} \cdot \vec{P} / \hbar} .
$$

Plugging these relations into the propagator, we obtain

$$
\begin{aligned}
K\left(q^{\prime \prime}, q^{\prime} ; t\right)= & \int_{\mathbb{R}_{\star}^{6}} \frac{d^{3} \vec{P} d^{3} \vec{R}}{(2 \pi \hbar / \ell)^{6}} e^{-\frac{i}{2 m \hbar} t \sum_{i} \mathcal{C}_{\star}} \star E_{k}(\vec{P}, \vec{R}) \\
& \star \int_{\mathrm{SU}(2)} d h E_{h}(\vec{P}, \vec{R}),
\end{aligned}
$$

where $(\vec{x}, \overrightarrow{\mathfrak{r}})=\left(\vec{x}\left(g^{\prime \prime} g^{\prime-1}\right), \overrightarrow{\mathfrak{r}}\left(g^{\prime \prime} g^{\prime-1}\right)\right)$. Again, the last term is just the delta function $\delta^{3}(\vec{R})$ on the rotation part (105), and we get, using the property (79) to eliminate the last $\star$-product,

$K\left(q^{\prime \prime}, q^{\prime} ; t\right)=\int_{\mathbb{R}_{\star}^{6}} \frac{d^{3} \vec{P} d^{3} \vec{R}}{(2 \pi \hbar / \ell)^{6}} \delta^{3}(\vec{R})\left(e_{\star}^{-\frac{i}{2 m \hbar} \mathcal{C}_{\star}} \star E_{k}(\vec{P}, \vec{R})\right)$.

Notice further that due to the $\star$ commutativity of the Casimir, the propagator can be rewritten in terms of a single $\star$-exponential as

$$
\begin{aligned}
& K\left(q^{\prime \prime}, q^{\prime} ; t\right)
\end{aligned}
$$

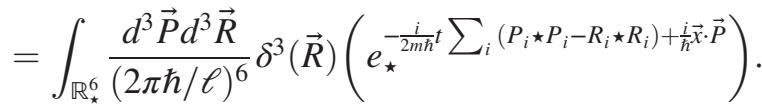

Expression (128) can be simplified noticing that from the properties of the Duflo map to preserve the algebra of invariant polynomials on $\mathfrak{g}$, the star-exponential involving the Casimir reduces to the standard exponential [see (B29)], and using also (126) we can rewrite the propagator as

$$
\begin{aligned}
K\left(q^{\prime \prime}, q^{\prime} ; t\right)= & \int_{\mathbb{R}_{\star}^{6}} \frac{d^{3} \vec{P} d^{3} \vec{R}}{(2 \pi \hbar / \ell)^{6}} \delta^{3}(\vec{R})\left(e^{-\frac{i}{2 m \hbar t} t\left(\vec{P}^{2}-\vec{R}^{2}+\frac{\hbar^{2}}{\ell^{2}}\right)} \star e^{i \vec{x} \cdot \vec{P} / \hbar}\right) \\
& \times \frac{(x / 2 \ell)^{2}}{\sinh ^{2}(x / 2 \ell)} .
\end{aligned}
$$

The expression for the propagator we have obtained, in its form (129) or (130), is the direct analogue of the commutative one, and presents the noncommutative algebra variables in the precise role we expect for the classical momenta in a path integral expression (here within a Fourier transform to configuration basis). This is the main result we wanted to show for this simple application of our formalism.

As a further remark, we point out that the propagator could be expressed in terms of only commutative momenta by calculating explicitly the $\star$-product in (130). We leave the calculation to a future work. We notice however for the interested reader that the calculation will necessarily result in some quantum correction to the classical expression. Considering that the action of the Casimir on a plane wave can be rephrased as the Laplacian in terms of Lie derivatives [see relations (8)], we expect that the quantum corrections arising from the $\star$-product can be encoded in an extra multiplicative term $\mathcal{K}\left(t \hbar /\left(m \ell^{2}\right), x^{2} / \ell^{2}\right)$ consisting is some combination of the dimensionless terms $t \hbar /\left(m \ell^{2}\right)$ and $x^{2} / \ell^{2}$, reducing to identity in the classical limit (which we take to be $\hbar \rightarrow 0$ followed by $\ell \rightarrow \infty$ ). In this case we can apply the delta function $\delta^{3}(R)$, and we are left with

$$
\begin{aligned}
K\left(q^{\prime \prime}, q^{\prime} ; t\right) \simeq & \int_{\mathbb{R}_{\star}^{3}} \frac{d^{3} \vec{P}}{(2 \pi \hbar / \ell)^{3}} e^{-\frac{i}{2 m \hbar} t\left(\vec{P}^{2}+\frac{\hbar^{2}}{\ell^{2}}\right)} e^{i \vec{x} \cdot \vec{P} / \hbar} \\
& \times \mathcal{K}\left(\frac{t \hbar}{m \ell^{2}}, \frac{x^{2}}{\ell^{2}}\right) \frac{(x / 2 \ell)^{2}}{\sinh ^{2}(x / 2 \ell)} .
\end{aligned}
$$

We can separate the radial and angular part of momenta $\vec{P}=P \hat{n}(P=|\vec{P}|)$ and, considering that the Casimir does not depend on the direction of $\vec{P}$, integrate the plane wave on the unit sphere to get

$$
\begin{aligned}
K\left(q^{\prime \prime}, q^{\prime} ; t\right) \simeq & \frac{1}{2 \pi^{2}} \int_{0}^{\infty} \frac{\ell}{\hbar} d P e^{-\frac{i}{2 m \hbar} t\left(P^{2}+\frac{\hbar^{2}}{\ell^{2}}\right)} \mathcal{K}\left(\frac{t \hbar}{m \ell^{2}}, \frac{x^{2}}{\ell^{2}}\right) \\
& \times \frac{\ell}{\hbar} P \frac{x\left(g^{\prime \prime} g^{\prime-1}\right) \sin \left(x\left(g^{\prime \prime} g^{\prime-1}\right) P / \hbar\right)}{2 \ell \sinh ^{2}\left(x\left(g^{\prime \prime} g^{\prime-1}\right) / \ell\right)},
\end{aligned}
$$

where we also wrote explicitly the dependence on the group element.

Comparing this result with the one for the propagator in the representation basis (117), we notice the similarity of the results, provided one uses the identification $r=\frac{\ell}{\hbar} P$. While the "classical" appearance of the propagator in the algebra representation would be maintained for a more complicated quantum system, e.g., a particle in an external 
potential and, more generally, with a more complicated action, in particular one depending on more than the quadratic invariant of the momenta, the similarity of the resulting expression with the expression in representation basis would be lost. This is to be expected, since harmonic functions are only eigenstates of the quadratic Casimir, while the states (120) are generalized eigenstates of all momentum operators. Finally, we can ascribe the correction terms in the exponential to the choice of (Duflo) quantization map (see the discussion of a similar term in the compact case in [49]).

To conclude our remark, we consider again expression (131)and take the infinitesimal propagator, for $t \rightarrow \epsilon$, $K\left(q^{\prime \prime}, q^{\prime} ; \epsilon\right)$. Expanding the factor $\mathcal{K}\left(\frac{\epsilon \hbar}{m \ell^{2}}, \frac{x^{2}}{\ell^{2}}\right)$ in powers of $\epsilon$ and $x^{2} / \ell^{2}$, we rewrite it as

$$
\mathcal{K}\left(\frac{\epsilon \hbar}{m \ell^{2}}, \frac{x^{2}}{\ell^{2}}\right) \simeq 1+\mathcal{K}_{0} \frac{x^{2}}{\ell^{2}}+O(\epsilon)+O\left(\frac{x^{4}}{\ell^{4}}\right),
$$

where $\mathcal{K}_{0}$ is some constant factor. Notice that, as expected in the algebra representation (54), we can rewrite functions of $\vec{x} / \ell$ as differential operators acting on the plane wave through the substitution $x^{i} \rightarrow-i \hbar \partial / \partial P_{i}$. We can thus integrate by part the functions involving those factors and, noticing also that the prefactor $\mathcal{A}(0, \vec{x} / \ell)$ is at least fourth order in $\vec{x} / \ell$, we obtain

$$
\begin{aligned}
& K\left(q^{\prime \prime}, q^{\prime} ; \epsilon\right) \\
& \quad=\int_{\mathbb{R}_{\star}^{3}} \frac{d^{3} \vec{P}}{(2 \pi \hbar / \ell)^{3}} e^{i \vec{x} \cdot \vec{P} / \hbar} e^{-\frac{i}{2 m \hbar} \epsilon\left(P^{2}+\frac{\hbar^{2}}{t^{2}}\left(1-6 \mathcal{K}_{0}\right)\right)}+O\left(\epsilon^{2}\right) .
\end{aligned}
$$

Making explicit its dependence on the group elements we can reexpress the infinitesimal propagator as

$$
\begin{aligned}
K\left(q^{\prime \prime}, q^{\prime} ; \epsilon\right) \simeq & \int_{\mathbb{R}^{3}} \frac{d^{3} P}{(2 \pi \hbar / \ell)^{3}} \exp \left(\frac { i } { \hbar } \epsilon \left(\frac{\vec{x}\left(g^{\prime \prime} g^{\prime-1}\right)}{\epsilon} \cdot \vec{P}\right.\right. \\
& \left.\left.-\frac{1}{2 m}\left(P^{2}+\frac{\hbar^{2}}{\ell^{2}}\left(1-6 \mathcal{K}_{0}\right)\right)\right)\right) .
\end{aligned}
$$

In the continuum limit, for the infinitesimal propagator we can rewrite $g^{\prime}=g(t), g^{\prime \prime}=g(t+\epsilon)$, so that $\left(\vec{x}\left(g g^{-1}\right)=0\right)$

$$
\frac{\vec{x}\left(g^{\prime-1} g^{\prime \prime}\right)}{\epsilon}=\vec{x}\left(g^{-1}(t) \frac{d g(t)}{d t}\right),
$$

which corresponds to the boost part of the canonical coordinates associated to the time derivative of the Maurer-Cartan form $\lambda=g^{-1} d g$, inducing the curved metric in the manifold $g_{a b}=\sum_{c} \lambda_{a}^{c} \lambda_{b}^{c}$. Denoting these coordinates as $x^{a}(g)=x^{i} \lambda_{i}^{a}(g)$ we thus finally get the finite time propagator

$$
\begin{aligned}
K\left(q^{\prime \prime}, q^{\prime} ; t\right)= & \int \mathcal{D} q \mathcal{D} P \exp \left(\frac { i } { \hbar } \int _ { t } ^ { t ^ { \prime } } d s \left(\dot{x}^{a}(g(s)) \vec{P}_{a}\right.\right. \\
& \left.\left.-\frac{1}{2 m}\left(P^{2}+\frac{\hbar^{2}}{\ell^{2}}\left(1-6 \mathcal{K}_{0}\right)\right)\right)\right) .
\end{aligned}
$$

The propagator takes the explicit form of a path-integral whose action appearing at the exponential coincides with the classical (Hamiltonian) action, confirming what we had anticipated looking at the infinitesimal propagator in the algebra representation. One can notice the quantum correction in the action [whose explicit form should be calculated solving the $\star$-product in (130)], as a shift in the energy, which characterizes the Duflo quantization map we have chosen. Similar corrections had been found in the Euclidean case, with a different quantization map, in [49].

\section{CONCLUSIONS}

We have defined a noncommutative algebra representation for quantum systems whose phase space is the cotangent bundle of the Lorentz group $T * \mathrm{SL}(2, \mathbb{C})$, and the noncommutative Fourier transform ensuring the unitary equivalence with the standard group representation. Our construction, following the general template presented in [15], is from first principles in the sense that all the structures are derived from the single initial input of a choice of quantization map for the classical system. Our specific construction corresponds to the choice of the Duflo quantization map, a choice motivated by the special mathematical properties of this map as well as by the interesting physical applications of the same, as we discussed in the text.

We have left all possible physical applications (beside the simple case of a point particle) of our construction aside, in this paper. However, we believe that our results could be of considerable impact in this direction. We have in mind in particular the application to quantum gravity, which can take two parallel paths. The first is in the context of model building for Lorentzian $4 \mathrm{~d}$ quantum gravity, within the group field theory and spin foam formalisms, so far quite limited (model building based on the Duflo map in the Riemannian context is discussed in [54]). In this context, Lorentzian model building should proceed alongside a more careful investigation of causal (or, better, "precausal") properties of the resulting models, possibly inspired by the early work [55]. The second is in the context of noncommutative spacetime field theories, where on the one hand our construction can offer a more mathematically solid ground for the construction of effective quantum gravity models, while on the other hand the possible phenomenological implications of the mathematical peculiarities of the Duflo map could be identified. 


\section{APPENDIX A: SOME PROPERTIES OF THE LORENTZ GROUP PARAMETRIZATION}

1. Explicit expression for the $S O(3,1)$ matrices and the relation between $\mathrm{SO}(3,1)$ and $\mathrm{SL}(2, \mathrm{C})$ representations

The explicit expression of $\Lambda(\vec{\rho}, \vec{\beta})$ can be obtained directly from (32) or evaluating the matrix exponential (38), and it is given by

$$
\begin{aligned}
\Lambda_{0}^{0}= & \frac{1}{\phi^{2}+\eta^{2}}\left(\frac{1}{2}\left(\phi^{2}+\eta^{2}+\vec{\rho}^{2}+\vec{\beta}^{2}\right) \cosh \eta\right. \\
& \left.+\frac{1}{2}\left(\phi^{2}+\eta^{2}-\vec{\rho}^{2}-\vec{\beta}^{2}\right) \cos \phi\right), \\
\Lambda_{i}^{0}= & \frac{1}{\phi^{2}+\eta^{2}}\left(-\rho_{i}(\phi \sinh \eta-\eta \sin \phi)\right. \\
& \left.-\beta_{i}(\eta \sinh \eta+\phi \sin \phi)+\epsilon_{i j k} \rho_{j} \beta_{k}(\cosh \eta-\cos \phi)\right), \\
\Lambda_{0}^{i}= & \frac{1}{\phi^{2}+\eta^{2}}\left(-\rho_{i}(\phi \sinh \eta-\eta \sin \phi)\right. \\
& \left.-\beta_{i}(\eta \sinh \eta+\phi \sin \phi)-\epsilon_{i j k} \rho_{j} \beta_{k}(\cosh \eta-\cos \phi)\right) \\
\Lambda_{j}^{i}= & \frac{1}{\phi^{2}+\eta^{2}}\left(\delta _ { i j } \left(\frac{1}{2}\left(\phi^{2}+\eta^{2}-\rho^{2}-\beta^{2}\right) \cosh \eta\right.\right. \\
& \left.+\frac{1}{2}\left(\phi^{2}+\eta^{2}+\rho^{2}+\beta^{2}\right) \cos \phi\right) \\
& +\left(\beta_{i} \beta_{j}+\rho_{i} \rho_{j}\right)(\cosh \eta-\cos \phi) \\
& +\epsilon_{i j k}\left(\beta_{k}(\phi \sinh \eta-\eta \sin \phi)\right. \\
& \left.\left.-\rho_{k}(\eta \sinh \eta+\phi \sin \phi)\right)\right), \\
&
\end{aligned}
$$

where the relation between $\phi, \eta, \vec{\rho}, \vec{\beta}$, are given by (30). This shows the relation between canonical coordinates on $\operatorname{SL}(2, \mathbb{C})$ and $\operatorname{SO}(3,1)$. As mentioned in the main text, the parameters domain is different for the two groups: the multivaluedness of the logarithmic map (the inverse of the exponential map) is determined by the periodicity of the compact subgroup of rotations. When $\eta=0 \quad(|\vec{\rho}|=\phi$, $\vec{\beta}=0)$, the group $\operatorname{SL}(2, \mathbb{C})$ [see (29)] reduces to $\operatorname{SU}(2)$ and the matrix $\Lambda$ represents a pure rotation

$$
\Lambda(\vec{\rho}, \vec{\beta}=0)=\left(\begin{array}{ll}
1 & 0_{3} \\
0_{3} & \mathcal{R}
\end{array}\right)
$$

where

$$
\mathcal{R}_{i j}=\delta_{i j} \cos \rho+\frac{\rho_{i} \rho_{j}}{\rho^{2}}(1-\cos \rho)-\epsilon_{i j k} \frac{\rho_{k}}{\rho} \sin \rho,
$$

So that

$$
\Lambda(|\vec{\rho}|=0, \vec{\beta}=0)=\Lambda(|\vec{\rho}|=2 \pi, \vec{\beta}=0)=\mathbb{1}_{4}
$$

While for the $\operatorname{SU}(2)$ subgroup of $\operatorname{SL}(2, \mathbb{C})$ we can see by setting $\eta=\vec{\beta}=0$ in (29) that

$a(\rho=0, \beta=0)=a(\rho=4 \pi, \beta=0)=\mathbb{1}_{2}$,

$a(\rho=2 \pi, \beta=0)=-\mathbb{1}_{2}$.

This shows that, analogously to the relation between $\mathrm{SO}(3)$ and $\mathrm{SU}(2), \mathrm{SL}(2, \mathbb{C})$ "covers twice" $\mathrm{SO}(3,1)$, manifesting the isomorphism $\mathrm{SO}(3,1) \simeq \operatorname{SL}(2, \mathbb{C}) /\{\mathbb{1},-\mathbb{1}\}$.

When $\phi=0+2 n \pi$ and $\eta \neq 0(\rho=0, \beta=\eta)$, the matrix $\Lambda$ represents a pure boost

$$
\Lambda(\vec{\rho}=0, \vec{\beta})=\left(\begin{array}{ll}
\mathcal{B}_{00} & \mathcal{B}_{0 i} \\
\mathcal{B}_{0 i} & \mathcal{B}_{i j}
\end{array}\right)
$$

where

$$
\begin{aligned}
\mathcal{B}_{00} & =\cosh \beta, \quad \mathcal{B}_{0 i}=-\frac{\beta_{i}}{\beta} \sinh \beta, \\
\mathcal{B}_{i j} & =\delta_{i j}+\frac{\beta_{i} \beta_{j}}{\vec{\beta}^{2}}(\cosh \beta-1) .
\end{aligned}
$$

\section{Branch cuts for the canonical coordinates}

When $\phi \neq 0+2 n \pi$ or $\eta \neq 0, a$ can be diagonalized and $\Lambda$ can be put in a normal form:

$$
\begin{aligned}
a & =a \delta a^{-1}, \delta=\left(\begin{array}{ccc}
e^{(\phi+i \eta) / 2} & 0 \\
0 & e^{-(\phi+i \eta) / 2}
\end{array}\right), \\
\Lambda(a) & =\Lambda(a) \Lambda(\delta) \Lambda\left(a^{-1}\right), \\
\Lambda(\delta) & =\left(\begin{array}{cccc}
\cosh \eta & 0 & 0 & \sinh \eta \\
0 & \cos \phi & \sin \phi & 0 \\
0 & -\sin \phi & \cos \phi & 0 \\
\sinh \eta & 0 & 0 & \cosh \eta
\end{array}\right) .
\end{aligned}
$$

Then $\Lambda$ belongs to an equivalent class of elements $\gamma(\phi, \eta)$ corresponding to the first of (26). If we restrict $0 \leq \phi<2 \pi$, each of these classes corresponds to a unique complex rotation angle, while one class $\gamma(\phi, \eta)$ of elements of $\Lambda \in \mathrm{SO}(3,1)$ corresponds to two classes $\gamma(\phi, \eta)$ and $\gamma(\phi+2 \pi, \eta)$ of elements of $\operatorname{SL}(2, \mathbb{C})$.

If $\phi=0+2 n \pi$ and $\eta=0$, so that $\vec{\zeta}^{2}=0$, and if also $\sum_{j}\left|\zeta_{j}\right|^{2}=0(\vec{\rho}=0, \vec{\beta}=0)$, then $a$ belongs to $\gamma(0,0)$. The second class in (26) can be obtained when $\phi=0, \eta=0$, so that $\vec{\zeta}^{2}=0$, but $\sum_{j}\left|\zeta_{j}\right|^{2}>0 \quad\left(\vec{\rho}^{2}=\vec{\beta}^{2}, \vec{\rho} \cdot \vec{\beta}=0\right.$ but $\left.\vec{\rho}^{2}+\vec{\beta}^{2}>0\right) .{ }^{16}$ The third class in (26) cannot be obtained

\footnotetext{
${ }^{16}$ For instance the representative of the second class in (26) can be obtained by setting $\beta_{1}=-1, \rho_{2}=1$ and all the other components to zero.
} 
[31,32] with the exponential map. Indeed the group $\mathrm{SL}(2, \mathbb{C})$ is not exponential. But one can show that it is weakly exponential. In order to do so consider the inverse map

$$
\zeta_{j}=\frac{2}{i} \frac{\log \left(a_{0}+\left(a_{0}^{2}-1\right)^{\frac{1}{2}}\right)}{\left(a_{0}^{2}-1\right)^{\frac{1}{2}}} a_{j} .
$$

The study of the branch points of this function shows that, restricting it to its principal values, the complex rotation vector is holomorphic in $a_{0}$ except for a branch cut extending on the real axis of $a_{0}$ from -1 to $-\infty$. Indeed the function in (A10) is single valued for all complex $a_{0}$. For $a_{0}$ real with $-1 \leq a_{0} \leq 1$ the numerator in (A10) is nothing but $\arccos \left(a_{0}\right)=\frac{1}{i} \log \left(a_{0}+i \sqrt{1-a_{0}^{2}}\right)$, which, extended to complex numbers, has branch cuts on the real axis for $a_{0}<-1$ and $a_{0}>1$. The interval $-1 \leq a_{0} \leq 1$ (and the imaginary axis of $a_{j}$ ) is realized by $\eta=0$, $\phi \in(-2 \pi, 2 \pi]$, i.e., by the $S U(2)$ subgroup of rotations. The half-line $a_{0}$ real with $a_{0}>1$ is taken by pure boosts $\phi=0, \quad \eta \neq 0$, and we can write the numerator as $\operatorname{arccosh}\left(a_{0}\right)=\log \left(a_{0}+\sqrt{a_{0}+1} \sqrt{a_{0}-1}\right)$, which, extended to complex $a_{0}$, has a branch cut on the real axis for $a_{0}<1$. The branch cut $a_{0}<-1$ is given by the composition of these two functions. The branch point $a_{0}=-1$ corresponds to the third class in (26). Thus, except for the branch cut, the canonical coordinates provided by the exponential map, represented by the complex rotation vector $\vec{\zeta}=\vec{\rho}+i \vec{\beta}$, are holomorphic functions parametrizing the whole $\mathrm{SL}(2, \mathbb{C})$ group.

\section{Haar measure for $\operatorname{SL}(2, \mathbb{C})$}

Considering an element

$$
a=\left(\begin{array}{ll}
\alpha & \beta \\
\gamma & \delta
\end{array}\right) \in \operatorname{SL}(2, \mathbb{C}),
$$

the Haar measure is defined $[29,47]$ as (the factor 2 is arbitrary)

$$
d g=\frac{2}{|\delta|^{2}} d \beta d \gamma d \delta d \beta^{*} d \gamma^{*} d \delta^{*},
$$

and has the invariance property

$$
d g=d(h g)=d(g h)=d g^{-1} .
$$

In the parametrization (23) evaluating the Jacobian

$$
\mathcal{J}\left(\left(\beta, \gamma, \delta, \beta^{*}, \gamma^{*}, \delta^{*}\right) \rightarrow\left(\vec{a}, \vec{a}^{*}\right)\right)=4\left|\frac{a_{3}-a_{0}}{a_{0}}\right|^{2}
$$

one gets the measure

$$
d g=\frac{8}{\left|a_{0}(\vec{a})\right|^{2}} d^{3} \vec{a} d^{3} \vec{a}^{*}
$$

where

$$
a_{0}(\vec{a})=\sqrt{1+\vec{a}^{2}} .
$$

The Jacobian to complex vector $\left(\zeta, \zeta^{*}\right)$ (28) can be evaluated to

$$
\mathcal{J}\left(\left(\vec{a}, \vec{a}^{*}\right) \rightarrow\left(\vec{\zeta}, \vec{\zeta}^{*}\right)\right)=\left|\frac{\sin \left(\frac{1}{2} \zeta\right) \sin (\zeta)}{4 \zeta^{2}}\right|^{2},
$$

from which

$$
d g=2 \frac{\left|\sin ^{4}\left(\frac{1}{2} \zeta\right)\right|}{\left|\zeta^{4}\right|} d^{3} \zeta d^{3} \zeta^{*}
$$

Finally, in terms of the real canonical coordinates $(\rho, \beta)(27)$ the Haar measure takes the form $\left(\mathcal{J}\left(\left(\zeta, \zeta^{*}\right) \rightarrow(\rho, \beta)\right)=8\right)$

$d g=\left(4 \frac{\cosh ^{2}\left(\frac{1}{2} \eta\right) \sin ^{2}\left(\frac{1}{2} \phi\right)+\sinh ^{2}\left(\frac{1}{2} \eta\right) \cos ^{2}\left(\frac{1}{2} \phi\right)}{\phi^{2}+\eta^{2}}\right)^{2} d^{3} \vec{\rho} d^{3} \vec{\beta}$.

Notice that the Haar measure in terms of canonical coordinates manifests the property (44) of the Duflo map.

\section{Haar measure in Cartan decomposition}

Considering the representation (27) and (92) we obtain the relations

$$
\begin{aligned}
\cos \left(\frac{1}{2} \zeta\right)= & \cosh \left(\frac{1}{2}|\overrightarrow{\mathfrak{b}}|\right) \cos \left(\frac{1}{2}|\overrightarrow{\mathfrak{r}}|\right) \\
& -i \sinh \left(\frac{1}{2}|\overrightarrow{\mathfrak{b}}|\right) \sin \left(\frac{1}{2}|\overrightarrow{\mathfrak{r}}|\right) \hat{\mathfrak{b}} \cdot \hat{\mathfrak{r}}, \\
\sin \left(\frac{1}{2} \zeta\right) \hat{\zeta}= & \cosh \left(\frac{1}{2}|\overrightarrow{\mathfrak{b}}|\right) \sin \left(\frac{1}{2}|\overrightarrow{\mathfrak{r}}|\right) \hat{\mathfrak{r}} \\
& +i \sinh \left(\frac{1}{2}|\overrightarrow{\mathfrak{b}}|\right) \cos \left(\frac{1}{2}|\overrightarrow{\mathfrak{r}}|\right) \hat{\mathfrak{b}} \\
& -i \sinh \left(\frac{1}{2}|\overrightarrow{\mathfrak{b}}|\right) \sin \left(\frac{1}{2}|\overrightarrow{\mathfrak{r}}|\right) \hat{\mathfrak{b}} \wedge \hat{\mathfrak{r}},
\end{aligned}
$$

where $\vec{\zeta}=\vec{\rho}+i \vec{\beta}$ and $\zeta^{2}=\vec{\zeta}^{2}=(\phi+i \eta)^{2}$. Equation (A20) is nothing but the Baker-Campbell-Hausdorff $(\mathrm{BCH})$ formula for the Cartan group element (92):

$g=\exp (i \vec{\rho} \cdot \vec{J}+i \vec{\beta} \cdot \vec{N})=\exp (i \overrightarrow{\mathfrak{b}} \cdot \vec{N}) \exp (i \overrightarrow{\mathfrak{r}} \cdot \vec{J})$ 
Defining the quantities

$$
\begin{aligned}
\vec{Z} & =\sin \left(\frac{1}{2} \zeta\right) \frac{\vec{\zeta}}{\zeta}, \\
\overrightarrow{\mathfrak{B}} & =\sinh \left(\frac{1}{2}|\overrightarrow{\mathfrak{b}}|\right) \hat{\mathfrak{b}}, \\
\overrightarrow{\mathfrak{R}} & =\sin \left(\frac{1}{2}|\overrightarrow{\mathfrak{r}}|\right) \hat{\mathfrak{r}},
\end{aligned}
$$

we get

$$
\begin{gathered}
\sqrt{1-\vec{Z}^{2}}=\sqrt{1+\overrightarrow{\mathfrak{B}}^{2}} \sqrt{1-\overrightarrow{\mathfrak{R}}^{2}}-i \overrightarrow{\mathfrak{B}} \cdot \overrightarrow{\mathfrak{R}} \\
\vec{Z}=\sqrt{1+\overrightarrow{\mathfrak{B}}^{2}} \overrightarrow{\mathfrak{R}}+i \sqrt{1-\overrightarrow{\mathfrak{R}}^{2}} \overrightarrow{\mathfrak{B}}+i \overrightarrow{\mathfrak{R}} \wedge \overrightarrow{\mathfrak{B}}
\end{gathered}
$$

In terms of the $\vec{Z}$ coordinates the measure is [see (A15) and consider that $\vec{a}=i \vec{Z}]$

$$
d g=\frac{8}{\left|\sqrt{1-\vec{Z}^{2}}\right|^{2}} d^{3} \vec{Z} d^{3} \vec{Z}^{*}
$$

The Jacobian of transformation from $\vec{Z}$ to $(\overrightarrow{\mathfrak{B}}, \overrightarrow{\mathfrak{R}})$ is

$$
\left|\frac{d^{3} \vec{Z} d^{3} \vec{Z}^{*}}{d^{3} \overrightarrow{\mathfrak{R}} d^{3} \overrightarrow{\mathfrak{B}}}\right|=8 \frac{\sqrt{1+\overrightarrow{\mathfrak{B}}^{2}}}{\sqrt{1-\overrightarrow{\mathfrak{R}}^{2}}}\left(\left(1-\overrightarrow{\mathfrak{R}}^{2}\right)\left(1+\overrightarrow{\mathfrak{B}}^{2}\right)+(\overrightarrow{\mathfrak{R}} \cdot \overrightarrow{\mathfrak{B}})^{2}\right) .
$$

The measure then becomes

$$
d g=64 \frac{\sqrt{1+\overrightarrow{\mathfrak{B}}^{2}}}{\sqrt{1-\overrightarrow{\mathfrak{R}}^{2}}} d^{3} \overrightarrow{\mathfrak{R}} d^{3} \overrightarrow{\mathfrak{B}}
$$

We can now rewrite it in terms of $\overrightarrow{\mathfrak{b}}$ and $\overrightarrow{\mathfrak{r}}$ coordinates. Considering the Jacobian of transformation (apart from numerical factors)

$$
\begin{aligned}
d^{3} \overrightarrow{\mathfrak{R}} & =\frac{\sin \left(\frac{1}{2}|\overrightarrow{\mathfrak{r}}|\right) \sin (|\overrightarrow{\mathfrak{r}}|)}{4|\overrightarrow{\mathfrak{r}}|^{2}} d^{3} \overrightarrow{\mathfrak{r}}, \\
d^{3} \overrightarrow{\mathfrak{B}} & =\frac{\sinh \left(\frac{1}{2}|\overrightarrow{\mathfrak{b}}|\right) \sinh (|\overrightarrow{\mathfrak{b}}|)}{4|\overrightarrow{\mathfrak{b}}|^{2}} d^{3} \overrightarrow{\mathfrak{b}}
\end{aligned}
$$

the measure becomes

$$
d g=4 \frac{\sinh ^{2}|\overrightarrow{\mathfrak{b}}|}{|\overrightarrow{\mathfrak{b}}|^{2}} \frac{\sin ^{2}\left(\frac{1}{2}|\overrightarrow{\mathfrak{r}}|\right)}{|\overrightarrow{\mathfrak{r}}|^{2}} d^{3} \overrightarrow{\mathfrak{r}} d^{3} \overrightarrow{\mathfrak{b}}
$$

\section{Delta on the group in canonical coordinates}

Consider that for canonical coordinates in $G$

$$
z(g h)=\mathcal{B}(z(g), z(h))
$$

where $\mathcal{B}(z(g), z(h))$ is the given by the $\mathrm{BCH}$ formula respect to the Lie algebra $\mathfrak{g}$. Indeed

$$
\begin{gathered}
g h=e^{i z(g) \cdot x} e^{i z(h) \cdot x}=e^{i \mathcal{B}(z(g), z(h)) \cdot x}, \\
g, h \in G, \quad x \in \mathfrak{g} .
\end{gathered}
$$

Since $z\left(g^{-1}\right)=-z(g), z(e)=0$,

$$
\mathcal{B}(z(g), z(h))=0 \quad \text { for } z(h)=-z(g) .
$$

The delta transforms with the inverse of the Jacobian of transformation

$$
\begin{aligned}
\delta(g h) & =\delta^{d}(\mathcal{B}(z(g), z(h))) \\
& =\left|\frac{d^{d} \mathcal{B}(z(g), z(h))}{d^{d} z(g)}\right|_{z(h)=-z(g)}^{-1} \delta^{d}(z(g)+z(h)) .
\end{aligned}
$$

But from the invariance of the Haar measure $d(g h)=d g$, so that

$$
d g=d^{d} z(g) \mu(z(g))=d^{d} z(g h) \mu(z(g h))
$$

it follows that the Jacobian is nothing but

$$
\left|\frac{d^{d} \mathcal{B}(z(g), z(h))}{d^{d} z(g)}\right|=\frac{\mu(z(g))}{\mu(\mathcal{B}(z(g), z(h)))},
$$

and we find, since $\mu(z(g)=0)=\mu(e)=1$,

$$
\delta(g h)=\mu^{-1}(z(g)) \delta^{d}(z(g)+z(h)) .
$$

\section{APPENDIX B: SOME CALCULATION FOR THE DUFLO QUANTIZATION MAP AND THE ASSOCIATED $\star$-PRODUCT}

\section{Calculation of the Duflo factor}

The Duflo function (43) can be evaluated explicitly making use of the identity

$$
\frac{\sinh \left(\frac{1}{2} x\right)}{\frac{1}{2} x}=\exp \left(\sum_{n \geq 1} \frac{B_{2 n}}{2 n(2 n) !} x^{2 n}\right)
$$

with $B_{2 n}$ Bernoulli numbers, so that, from the property of the determinant

$$
\operatorname{det}(\exp (A))=\exp (\operatorname{Tr} A)
$$

we get 


$$
j(x)=\exp \left(\sum_{n \geq 1} \frac{B_{2 n}}{2 n(2 n) !} \operatorname{Tr}\left(\operatorname{ad}_{x}\right)^{2 n}\right) .
$$

In order to simplify the calculations we adopt the following standard redefinition of the Lorentz generators:

$$
\vec{L}=\frac{1}{2}(\vec{J}-i \vec{N}), \quad \vec{R}=\frac{1}{2}(\vec{J}+i \vec{N}),
$$

satisfying the brackets

$$
\left[L_{i}, L_{j}\right]=i \epsilon_{i j k} L_{k}, \quad\left[R_{i}, R_{j}\right]=i \epsilon_{i j k} R_{k}, \quad\left[L_{i}, R_{j}\right]=0 .
$$

We have thus split the algebra in two mutually commuting sets of $\mathfrak{g} \mathfrak{u}(2)$ generators $L_{i}$ and $R_{i}$, in terms of which a group element (40) takes the form

$$
g=\exp (i \vec{\zeta} \cdot \vec{L}) \exp \left(i \vec{\zeta}^{*} \cdot \vec{R}\right)
$$

where $\zeta_{i}=\rho_{i}+i \beta_{i}$ as above. Defining a generic element of $\mathfrak{g}$ in this basis as $x=x^{I} E_{I}=x_{L}^{i} L_{i}+x_{R}^{i} R_{i}$, for $I=1, \ldots, 6$, with $E_{I}=L_{I}$ for $I=1,2,3, E_{I}=R_{I-3}$ for $I=4,5,6$, the adjoint representation is given by the matrix $\left(\operatorname{ad}_{X}\right)_{J}^{K}=x^{I} c_{I J}{ }^{K}$, where $c_{I J}{ }^{K}$ are the structure constant of $\mathfrak{g}$ given by (B5) as $c_{I J}{ }^{K}=i \epsilon_{I J}{ }^{K}$ for $I, J, K=1,2,3$, while $c_{I J}{ }^{K}=i \epsilon_{I-3 J-3}^{K-3}$ for $I, J, K=4,5,6$. It follows by direct computation that

$$
\begin{aligned}
\operatorname{ad}_{X} \operatorname{ad}_{Y} & =\left\{\left(\operatorname{ad}_{x}\right)_{J}^{I}\left(\operatorname{ad}_{y}\right)_{I}^{K}\right\} \\
& =\left(\begin{array}{cc}
\left\{\delta_{j}^{k} \vec{x}_{L} \cdot \vec{y}_{L}-x_{L j} y_{L}^{k}\right\} & \mathbf{0}_{3 \times 3} \\
\mathbf{0}_{3 \times 3} & \left\{\delta_{j}^{k} \vec{x}_{R} \cdot \vec{y}_{R}-x_{R j} y_{R}^{k}\right\}
\end{array}\right),
\end{aligned}
$$

so that, with the notation $x=\sqrt{\vec{x} \cdot \vec{x}}(=|\vec{x}|$ if $\vec{x}$ is real $)$

$$
\operatorname{Tr}\left(\operatorname{ad}_{x} \operatorname{ad}_{x}\right)=2 x^{2}=2 x_{L}^{2}+2 x_{R}^{2} .
$$

With a similar calculation one finds that

$$
\operatorname{Tr}\left(\operatorname{ad}_{x}^{2 n}\right)=2 x_{L}^{2 n}+2 x_{R}^{2 n},
$$

and finally

$$
\begin{aligned}
j(x) & =\exp \left(2 \sum_{n \geq 1} \frac{B_{2 n}}{2 n(2 n) !}\left(x_{L}^{2 n}+x_{\mathrm{R}}^{2 n}\right)\right) \\
& =\exp \left(2 \sum_{n \geq 1} \frac{B_{2 n}}{2 n(2 n) !} x_{L}^{2 n}\right) \exp \left(2 \sum_{n \geq 1} \frac{B_{2 n}}{2 n(2 n) !} x_{R}^{2 n}\right) \\
& =16 \frac{\sinh ^{2}\left(\frac{1}{2} x_{L}\right)}{x_{L}^{2}} \frac{\sinh ^{2}\left(\frac{1}{2} x_{R}\right)}{x_{R}^{2}} .
\end{aligned}
$$

We can now rewrite the Duflo factor in the canonical basis generated by $e_{i} \equiv\left(J_{i}, N_{i}\right)$ [for which we have canonical coordinates $\left.k^{i} \equiv\left(\rho^{i}, \beta^{i}\right)\right]$. Considering that an element of the Lie algebra is $x=x_{L}^{i} L_{i}+x_{R}^{i} R_{i}=x_{J}^{i} J_{i}+x_{N}^{i} N_{i}$, we get

$$
x_{L}^{i}=x_{J}^{i}+i x_{N}^{i}, \quad x_{R}^{i}=x_{J}^{i}-i x_{N}^{i},
$$

and

$$
j^{\frac{1}{2}}(x)=4 \frac{\left|\sinh \left(\frac{1}{2} x_{\zeta}\right)\right|^{2}}{\left|x_{\zeta}^{2}\right|},
$$

where $x_{\zeta}=\sqrt{\vec{x}_{\zeta} \cdot \vec{x}_{\zeta}}$ and $x_{\zeta}^{i}=x_{J}^{i}+i x_{N}^{i}$.

Notice that if we rewrite the exponential function on $\mathfrak{g}^{*}$ as

$\exp \left(i k_{I} X^{I}\right)=\exp \left(i \vec{\zeta} \cdot \vec{X}_{L}\right) \exp \left(i \vec{\zeta}^{*} \cdot \vec{X}_{R}\right) \quad X^{I} \in \mathfrak{g}^{*}$

where in this basis the coordinates on $\mathfrak{g}^{*}$ are $\mathrm{e}^{17}$

$$
X_{i}^{L}=\frac{1}{2}\left(X_{i}^{J}-i X_{i}^{N}\right), \quad X_{i}^{R}=\frac{1}{2}\left(X_{i}^{J}+i X_{i}^{N}\right),
$$

we can also apply the Duflo function directly on the form (B10), i.e., apply the function $j^{\frac{1}{2}}(\partial)$ to the exponential (B13) to get

$$
\left(j^{\frac{1}{2}}(\partial) \exp \right)\left(i k^{I} X_{I}\right)=4 \frac{\left|\sin \left(\frac{1}{2} \zeta\right)\right|^{2}}{\left|\zeta^{2}\right|} \exp \left(i k^{I} X_{I}\right)
$$

which coincides with (48).

\section{Explicit calculation of the $\star$-product on monomials}

Considering expressions (18) and (19), the $\star$-product between $n$ coordinates of $\mathfrak{g}^{*}$ can be evaluated through the formula

$$
\begin{aligned}
X_{i_{1}} \star & X_{i_{2}} \star \cdots X_{i_{n}} \\
= & \left.(-i)^{n} \frac{\partial^{n}}{\partial k_{1}^{i_{1}} \partial k_{2}^{j_{2}} \cdots \partial k_{3}^{i_{n}}}\right|_{k_{1}=k_{2}=\cdots=k_{n}=0} \\
& \times \mathcal{D}^{-1}\left(e^{i \overrightarrow{\mathcal{B}}\left(k_{1}, k_{2}, \ldots, k_{n}\right) \cdot \hat{X}}\right) .
\end{aligned}
$$

The term to differentiate in the last expression can be rewritten as

$$
\mathcal{A}\left(\mathcal{B}\left(k_{1}, k_{2}, \ldots, k_{n}\right)\right) \mathcal{S}^{-1}\left(e^{i \vec{k}_{1} \cdot \hat{X}} e^{i \vec{k}_{2} \cdot \hat{X}} \cdots e^{i \vec{k}_{1} \cdot \hat{X}}\right) .
$$

For the lowest order powers for the $\star$-product we get

\footnotetext{
${ }^{17}$ This can be seen using the duality relations $\left\langle e_{i}, \tilde{e}_{j}\right\rangle=\delta_{i j}$ from which $\langle x, X\rangle=x_{J}^{i} X_{i}^{J}+x_{N}^{i} X_{i}^{N}=x_{L}^{i} X_{i}^{L}+x_{R}^{i} X_{i}^{R}$.
} 


$$
X_{i} \star X_{j}=\left.\frac{(-i)^{2} \partial^{2}}{\partial k_{1}^{i} \partial k_{2}^{j}}\right|_{k=0}\left(\mathcal{A}_{k\left(k_{1}, k_{2}\right)}^{(2)}\left(k_{1}, k_{2}\right)+i^{2} \vec{k}_{1}^{i} \vec{k}_{2}^{j} \mathcal{S}^{-1}\left(\hat{X}_{i} \hat{X}_{j}\right)\right),
$$

$$
\begin{aligned}
X_{i} \star X_{j} \star X_{k}= & \left.\frac{(-i)^{3} \partial^{3}}{\partial k_{1}^{i} \partial k_{2}^{j} \partial k_{3}^{k}}\right|_{k=0}\left(\mathcal{A}_{k\left(k_{1}, k_{2}, k_{3}\right)}^{(3)}\left(k_{1}, k_{2}, k_{3}\right)\right. \\
& +i^{3} k_{1}^{m} k_{2}^{n} k_{3}^{l} \mathcal{S}^{-1}\left(\hat{X}_{m} \hat{X}_{n} \hat{X}_{l}\right) \\
& +i\left(\mathcal{A}_{k\left(k_{1}, k_{2}, k_{3}\right)}^{(2)}\left(k_{1}, k_{2}\right) k_{3}^{l}\right. \\
& +\mathcal{A}_{k\left(k_{1}, k_{2}, k_{3}\right)}^{(2)}\left(k_{1}, k_{3}\right) k_{2}^{l} \\
& \left.\left.+\mathcal{A}_{k\left(k_{1}, k_{2}, k_{3}\right)}^{(2)}\left(k_{2}, k_{3}\right) k_{1}^{l}\right) X_{l}\right),
\end{aligned}
$$

where we indicated with $\mathcal{A}_{k\left(k_{1}, k_{2}, \ldots, k_{n}\right)}^{(i)}\left(k_{a}, k_{b}, \ldots, k_{c}\right)$ the (i)-order mixed term in $k_{a}, k_{b}, \ldots, k_{c}$ of the $n$-ple Duflo factor.

In terms of the complex vector $\vec{\zeta}=\vec{\rho}+i \vec{\beta}$, defining $\overrightarrow{\mathcal{B}}\left(k_{1}, k_{2}, \ldots, k_{n}\right)=\vec{\zeta}\left(\zeta_{1}, \zeta_{2}, \ldots, \zeta_{n}\right)$, we can calculate the $\mathrm{BCH}$ formula from the $\mathrm{SL}(2, \mathrm{C})$ representation (28)

$$
e^{\frac{i}{\zeta} \vec{\zeta} \cdot \sigma}=\cos \left(\frac{1}{2} \vec{\zeta} \cdot \sigma\right) \mathbb{1}+i \sin \left(\frac{1}{2} \vec{\zeta} \cdot \sigma\right) \hat{\zeta} \cdot \sigma
$$

from which follows for the double $\mathrm{BCH}$ (the $\mathrm{BCH}$ coming from the product of two exponentials)

$$
\tan \left(\frac{1}{2} \zeta\left(\zeta_{1}, \zeta_{2}\right)\right) \hat{\zeta}\left(\zeta_{1}, \zeta_{2}\right)=\frac{\sin \left(\frac{1}{2} \zeta_{1}\right) \cos \left(\frac{1}{2} \zeta_{2}\right) \hat{\zeta}_{1}+\cos \left(\frac{1}{2} \zeta_{1}\right) \sin \left(\frac{1}{2} \zeta_{2}\right) \hat{\zeta}_{2}-\sin \left(\frac{1}{2} \zeta_{1}\right) \sin \left(\frac{1}{2} \zeta_{2}\right) \hat{\zeta}_{1} \wedge \hat{\zeta}_{2}}{\cos \left(\frac{1}{2} \zeta_{1}\right) \cos \left(\frac{1}{2} \zeta_{2}\right)-\sin \left(\frac{1}{2} \zeta_{1}\right) \sin \left(\frac{1}{2} \zeta_{2}\right) \hat{\zeta}_{1} \cdot \hat{\zeta}_{2}}
$$

The Duflo factor (52) [or inverse (48)] can be expanded as $(\{k\}=\{\zeta\}$ or $\{\rho, \beta\})$

$$
\begin{aligned}
\mathcal{A} & =1+\frac{1}{24}\left(\zeta^{2}+\zeta^{* 2}\right)+O\left(k^{4}\right) \\
& =1+\frac{1}{12}\left(\rho^{2}-\beta^{2}\right)+O\left(k^{4}\right) .
\end{aligned}
$$

Since the BCH (B21) for $\vec{\zeta}$ is at least linear in $\zeta_{1}$ and $\zeta_{2}$, it is enough to consider the expansion of the $\mathrm{BCH}$ up to 2 nd order in the coordinates $\{k\}$

$$
\vec{\zeta}\left(\zeta_{1}, \zeta_{2}\right) \simeq \vec{\zeta}_{1}+\vec{\zeta}_{2}-\frac{1}{2} \vec{\zeta}_{1} \wedge \vec{\zeta}_{2}
$$

or

$$
\begin{aligned}
& \vec{\rho}\left(\rho_{1}, \rho_{2}\right) \simeq \vec{\rho}_{1}+\vec{\rho}_{2}-\frac{1}{2}\left(\vec{\rho}_{1} \wedge \vec{\rho}_{2}-\vec{\beta}_{1} \wedge \vec{\beta}_{2}\right), \\
& \vec{\beta}\left(\beta_{1}, \beta_{2}\right) \simeq \vec{\beta}_{1}+\vec{\beta}_{2}-\frac{1}{2}\left(\vec{\rho}_{1} \wedge \vec{\beta}_{2}+\vec{\beta}_{1} \wedge \vec{\rho}_{2}\right) .
\end{aligned}
$$

For the triple $\mathrm{BCH}$ we can use the associativity ${ }^{18}$ property $\mathcal{B}\left(\zeta_{1}, \zeta_{2}, \zeta_{3}\right)=\mathcal{B}\left(\zeta_{1}, \mathcal{B}\left(\zeta_{2}, \zeta_{3}\right)\right)$, from which we get, up to 2nd order

$$
\begin{aligned}
\vec{\rho}\left(\rho_{1}, \rho_{2}, \rho_{2}\right) \simeq & \vec{\rho}_{1}+\vec{\rho}_{2}+\vec{\rho}_{3}-\frac{1}{2}\left(\vec{\rho}_{1} \wedge \vec{\rho}_{2}+\vec{\rho}_{1} \wedge \vec{\rho}_{3}\right. \\
& \left.+\vec{\rho}_{2} \wedge \vec{\rho}_{3}-\vec{\beta}_{1} \wedge \vec{\beta}_{2}-\vec{\beta}_{1} \wedge \vec{\beta}_{3}-\vec{\beta}_{2} \wedge \vec{\beta}_{3}\right), \\
\vec{\beta}\left(\beta_{1}, \beta_{2}, \beta_{2}\right) \simeq & \vec{\beta}_{1}+\vec{\beta}_{2}+\vec{\beta}_{3}-\frac{1}{2}\left(\vec{\rho}_{1} \wedge \vec{\beta}_{2}+\vec{\rho}_{1} \wedge \vec{\beta}_{3}\right. \\
& \left.+\vec{\rho}_{2} \wedge \vec{\beta}_{3}+\vec{\beta}_{1} \wedge \vec{\rho}_{2}+\vec{\beta}_{1} \wedge \vec{\rho}_{3}+\vec{\beta}_{2} \wedge \vec{\rho}_{3}\right) .
\end{aligned}
$$

The symmetrization map (42) on 2nd and 3rd order monomials is such that

\footnotetext{
${ }^{18}$ The associativity of the $\mathrm{BCH}$ comes from the associativity of the group product.
}

$$
\begin{aligned}
& \mathcal{S}^{-1}\left(\hat{X}_{i} \hat{X}_{j}\right)=X_{i} X_{j}+\frac{1}{2} \mathcal{S}^{-1}\left(\left[\hat{X}_{i}, \hat{X}_{j}\right]\right), \\
& S^{-1}\left(\hat{X}_{i} \hat{X}_{j} \hat{X}_{k}\right)= X_{i} X_{j} X_{k}+\frac{1}{2}\left(X_{i} \mathcal{S}^{-1}\left(\left[\hat{X}_{j}, \hat{X}_{k}\right]\right)\right. \\
&\left.+X_{j} S^{-1}\left(\left[\hat{X}_{i}, \hat{X}_{k}\right]\right)+X_{k} \mathcal{S}^{-1}\left(\left[\hat{X}_{i}, \hat{X}_{j}\right]\right)\right) \\
&+\frac{1}{12} \mathcal{S}^{-1}\left(3\left[\hat{X}_{i},\left[\hat{X}_{j}, \hat{X}_{k}\right]\right]\right. \\
&\left.-\left[\hat{X}_{j},\left[\hat{X}_{i}, \hat{X}_{k}\right]\right]-\left[\hat{X}_{k},\left[\hat{X}_{i}, \hat{X}_{j}\right]\right]\right)
\end{aligned}
$$

Substituting (B24), (B25) in (B22), and using the commutation relations (55) we can finally calculate from (B18) and (B19). We here report the explicit expression of third order monomials for the Duflo-map $\star$-product (repeated indexes are summed):

$$
\begin{aligned}
X_{i}^{J} \star X_{j}^{J} \star X_{k}^{J}= & X_{i}^{J} X_{j}^{J} X_{k}^{J}+\frac{i}{2}\left(\epsilon_{i j}^{l} X_{k}^{J} X_{l}^{J}+\epsilon_{i k}^{l} X_{j}^{J} X_{l}^{J}\right. \\
& \left.+\epsilon_{j k}^{l} X_{i}^{J} X_{l}^{J}\right)-\frac{1}{2} \delta_{i k} X_{j}^{J}-\frac{i}{12} \epsilon_{i j k}, \\
X_{i}^{J} \star X_{j}^{J} \star X_{k}^{N}= & X_{i}^{J} X_{j}^{J} X_{k}^{N}+\frac{i}{2}\left(\epsilon_{i j}^{l} X_{k}^{N} X_{l}^{J}+\epsilon_{i k}^{l} X_{j}^{J} X_{l}^{N}\right. \\
& \left.+\epsilon_{j k}^{l} X_{i}^{J} X_{l}^{N}\right)+\frac{1}{6}\left(\delta_{j k} X_{i}^{N}-2 \delta_{i k} X_{j}^{N}\right), \\
X_{i}^{N} \star X_{j}^{N} \star X_{k}^{N}= & X_{i}^{N} X_{j}^{N} X_{k}^{N}-\frac{i}{2}\left(\epsilon_{i j}^{l} X_{k}^{N} X_{l}^{J}+\epsilon_{i k}^{l} X_{j}^{N} X_{l}^{J}\right. \\
& \left.+\epsilon_{j k}^{l} X_{i}^{N} X_{l}^{J}\right)+\frac{1}{2}\left(\delta_{i k} X_{j}^{N}\right), \\
X_{i}^{N} \star X_{j}^{N} \star X_{k}^{J}= & X_{i}^{N} X_{j}^{N} X_{k}^{J}-\frac{i}{2}\left(\epsilon_{i j}^{l} X_{k}^{J} X_{l}^{J}-\epsilon_{i k}^{l} X_{j}^{N} X_{l}^{N}\right. \\
& \left.-\epsilon_{j k}^{l} X_{i}^{N} X_{l}^{N}\right)+\frac{1}{6}\left(2 \delta_{i k} X_{j}^{J}-\delta_{j k} X_{i}^{J}\right)+\frac{i}{12} \epsilon_{i j k} .
\end{aligned}
$$


From the properties of the Duflo map it is also easy to prove that the Duflo star product preserves the algebra of $\operatorname{Sym}(\mathfrak{g})^{\mathfrak{g}}$. One can check explicitly for instance that given the quadratic Casimir $\hat{\mathcal{C}}=\hat{X}_{J}^{2}-\hat{X}_{N}^{2}$ of the quantum algebra, such that

$\mathcal{C}_{\star}=\mathcal{D}^{-1}(\hat{\mathcal{C}})=X_{i}^{J} \star X_{i}^{J}-X_{i}^{N} \star X_{i}^{N}=\vec{X}_{J}^{2}-\vec{X}_{N}^{2}-1$,

$$
\hat{\mathcal{C}} \cdot \hat{\mathcal{C}}=\mathcal{D}\left(\mathcal{C}_{\star}\right) \cdot \mathcal{D}\left(\mathcal{C}_{\star}\right)=\mathcal{D}\left(\mathcal{C}_{\star}^{2}\right),
$$

i.e.,

$$
\mathcal{C}_{\star} \star \mathcal{C}_{\star}=\mathcal{C}_{\star}^{2}
$$

\section{APPENDIX C: SOME PROPERTIES OF THE HOMOGENEOUS SPACE $\mathcal{H}_{3} \approx \mathrm{SL}(2, \mathbb{C}) / \mathrm{SU}(2)$}

\section{Hyperbolic rotation angle}

We show in this subsection the relation between the Cartan parametrization of Sec. IV D and the parametrization in terms of "hyperbolic rotations" used in [50,51]. These can be considered as the generalization to hyperbolic space of Euler angles rotations. The only change respect to the Cartan splitting of Sec. IV D is in the "boost" sector $\mathrm{SL}(2, \mathbb{C}) / \mathrm{SU}(2) \quad[$ or $\mathrm{SO}(3,1) / \mathrm{SO}(3)$ ]. In this case an element of $\operatorname{SL}(2, \mathbb{C})$ [or $\mathrm{SO}(3,1)]$ is split as

$$
g=\mathcal{K} h,
$$

where $h \in \mathrm{SU}(2)$ [or $\mathrm{SO}(3)$ ], and $\mathcal{K} \in \mathrm{SL}(2, \mathbb{C}) / \mathrm{SU}(2)$ [or $\mathrm{SO}(3,1) / \mathrm{SO}(3)]$ such that

$$
\mathcal{K}=h_{1}\left(\alpha_{1}\right) h_{3}\left(\alpha_{3}\right) \mathcal{K}_{3}(\Theta),
$$

where $h_{1}\left(\alpha_{1}\right)$ and $h_{3}\left(\alpha_{3}\right)$ are Euclidean rotations around respectively the 1 and 3 axes, while $\mathcal{K}_{3}(\Theta)$ is an hyperbolic rotation (a boost) along the 3 axis:

$$
\mathcal{K}_{3}(\Theta)=\left\{\begin{array}{cccc}
\cosh \left(\frac{1}{2} \Theta\right) \mathbb{1}_{2} & -\sinh \left(\frac{1}{2} \Theta\right) \sigma_{3} & \text { in } \operatorname{SL}(2, \mathbb{C}) / \operatorname{SU}(2), \\
\cosh (\Theta) & 0 & 0 & \sinh (\Theta) \\
0 & 1 & 0 & 0 \\
0 & 0 & 1 & 0 \\
\sinh (\Theta) & 0 & 0 & \cosh (\Theta)
\end{array}\right) \text { in } \operatorname{SO}(3,1) / \operatorname{SO}(3) .
$$

The action of $\mathcal{K}$ on the origin $q_{a}=(1, \overrightarrow{0})$ of $\mathcal{H}_{3}$ gives a generic point of $q=\mathcal{K} q_{a} \mathcal{K}^{\dagger}\left(q=\mathcal{K} q_{a}\right.$ for $\left.\mathrm{SO}(3,1) / \mathrm{SO}(3)\right)$ of $\mathcal{H}_{3}$ as

$$
\begin{aligned}
& q_{0}=\cosh (\Theta), \\
& q_{1}=\sinh (\Theta) \sin \left(\alpha_{3}\right) \sin \left(\alpha_{1}\right), \\
& q_{2}=\sinh (\Theta) \sin \left(\alpha_{3}\right) \cos \left(\alpha_{1}\right), \\
& q_{3}=\sinh (\Theta) \cos \left(\alpha_{3}\right) .
\end{aligned}
$$

By comparison with Eq. (94) we find that

$$
\begin{aligned}
\Theta & =\mathfrak{b}, \\
\hat{\mathfrak{b}} & =\left(\sin \left(\alpha_{3}\right) \sin \left(\alpha_{1}\right), \sin \left(\alpha_{3}\right) \cos \left(\alpha_{1}\right), \cos \left(\alpha_{3}\right)\right) .
\end{aligned}
$$

Moreover, considering the Jacobian

$$
d^{3} \overrightarrow{\mathfrak{b}}=\Theta^{2} \sin \left(\alpha_{1}\right) d \Theta d \alpha_{1} d \alpha_{3},
$$

the measure (97) becomes

$$
d g=\sinh ^{2}(\Theta) \sin \left(\alpha_{1}\right) d \Theta d \alpha_{1} d \alpha_{3} d h .
$$

\section{Projection to $\mathcal{H}_{3}$ of the propagator}

The propagator in $\mathcal{H}_{3}$ can be rewritten as

$$
\begin{aligned}
K\left(q^{\prime \prime}, q^{\prime} ; t\right)= & \left\langle q^{\prime \prime}|U(t)| q^{\prime}\right\rangle \\
= & \int_{\operatorname{SL}(2, \mathbb{C})} d g^{\prime} \\
& \times \int_{\operatorname{SL}(2, \mathbb{C})} d g^{\prime \prime}\left\langle q^{\prime \prime} \mid g^{\prime \prime}\right\rangle\left\langle g^{\prime \prime}|U(t)| g^{\prime}\right\rangle\left\langle g^{\prime} \mid q^{\prime}\right\rangle .
\end{aligned}
$$

Defining

$$
\left\langle g^{\prime} \mid q^{\prime}\right\rangle\left\langle q^{\prime \prime} \mid g^{\prime \prime}\right\rangle=\delta\left(q^{\prime} k^{\prime-1}\right) \delta\left(q^{\prime \prime} k^{\prime \prime-1}\right) \delta\left(h^{\prime} h^{\prime \prime-1}\right)
$$

for the Cartan splitting (92) $g^{\prime}=k^{\prime} h^{\prime}, g^{\prime \prime}=k^{\prime \prime} h^{\prime \prime}$, we get

$$
\begin{aligned}
& K\left(q^{\prime \prime}, q^{\prime} ; t\right)=\int_{\mathcal{H}_{3}} d k^{\prime} \int_{\mathrm{SU}(2)} d h^{\prime} \int_{\mathcal{H}_{3}} d k^{\prime \prime} \\
& \times \int_{\mathrm{SU}(2)} d h^{\prime \prime} \delta\left(q^{\prime} k^{\prime-1}\right) \delta\left(q^{\prime \prime} k^{\prime \prime-1}\right) \\
& \times \delta\left(h^{\prime} h^{\prime \prime-1}\right)\left\langle g^{\prime \prime}|U(t)| g^{\prime}\right\rangle \\
& =\int_{\mathrm{SU}(2)} d h\left\langle q^{\prime \prime} h|U(t)| q^{\prime} h\right\rangle, \\
& \text { i.e., } \\
& \begin{aligned}
K\left(q^{\prime \prime}, q^{\prime} ; t\right) & =\int_{\mathrm{SU}(2)} d h K_{G}\left(q^{\prime \prime} h, q^{\prime} h ; t\right) \\
& =\int_{\mathrm{SU}(2)} d h K_{G}\left(g=g^{\prime \prime} g^{\prime-1} ; t\right)
\end{aligned}
\end{aligned}
$$

where we also used the invariance of the SU(2) Haar measure $d\left(h h^{\prime}\right)=d h$. 
[1] M. D. Ciric, N. Konjik,M. Kurkov, F. Lizzi, and P. Vitale, Noncommutative field theory from angular twist, Phys. Rev. D 98, 085011 (2018); H. Grosse and R. Wulkenhaar, Noncommutative quantum field theory, Fortschr. Phys. 62, 797 (2014); R. Szabo, Quantum gravity, field theory and signatures of noncommutative spacetime, Gen. Relativ. Gravit. 42, 1 (2010).

[2] S. Hossenfelder, Minimal length scale scenarios for quantum gravity, Living Rev. Relativity 16, 2 (2013); S. Liberati and L. Maccione, Quantum gravity phenomenology: Achievements and challenges, J. Phys. Conf. Ser. 314, 012007 (2011); G. Amelino-Camelia, Quantum gravity phenomenology, Living Rev. Relativity 16, 5 (2013).

[3] S. Hossenfelder, Bounds on an Energy-Dependent and Observer-Independent Speed of Light from Violations of Locality, Phys. Rev. Lett. 104, 140402 (2010).

[4] G. Amelino-Camelia, M. Matassa, F. Mercati, and G. Rosati, Taming Nonlocality in Theories with Planck-Scale Deformed Lorentz Symmetry, Phys. Rev. Lett. 106, 071301 (2011).

[5] G. Amelino-Camelia, L. Freidel, J. Kowalski-Glikman, and L. Smolin, The principle of relative locality, Phys. Rev. D 84, 084010 (2011).

[6] L. Freidel, J. Kowalski-Glikman, and S. Nowak, From noncommutative kappa-Minkowski to Minkowski spacetime, Phys. Lett. B 648, 70 (2007); Field theory on kappaMinkowski space revisited: Noether charges and breaking of Lorentz symmetry, Int. J. Mod. Phys. A 23, 2687 (2008).

[7] S. Majid and R. Oeckl, Twisting of quantum differentials and the Planck scale Hopf algebra, Commun. Math. Phys. 205, 617 (1999).

[8] P. Kosinski, J. Lukierski, and P. Maslanka, Local $D=4$ field theory on kappa deformed Minkowski space, Phys. Rev. D 62, 025004 (2000).

[9] G. Amelino-Camelia and S. Majid, Waves on noncommutative space-time and gamma-ray bursts, Int. J. Mod. Phys. A 15, 4301 (2000).

[10] D. Oriti, in Foundations of Space and Time, edited by G. Ellis, J. Murugan, and A. Weltman (Cambridge University Press, Cambridge, England, 2012); T. Krajewski, Group field theories, Proc. Sci., QGQGS2011 (2011) 005; D. Oriti, in Loop Quantum Gravity, edited by A. Ashtekar and J. Pullin (World Scientific, Singapore, 2017).

[11] A. Perez, The spin-foam approach to quantum gravity, Living Rev. Relativity 16, 3 (2013).

[12] A. Ashtekar and J. Lewandowski, Background independent quantum gravity: A status report, Classical Quantum Gravity $\mathbf{2 1}$, R53 (2004).

[13] A. Baratin and D. Oriti, Group Field Theory with Noncommutative Metric Variables, Phys. Rev. Lett. 105, 221302 (2010); Group field theory and simplicial gravity path integrals: A model for Holst-Plebanski gravity, Phys. Rev. D 85, 044003 (2012); A. Baratin, F. Girelli, and D. Oriti, Diffeomorphisms in group field theories, Phys. Rev. D 83, 104051 (2011).

[14] A. Baratin, B. Dittrich, D. Oriti, and J. Tambornino, commutative flux representation for loop quantum gravity, Classical Quantum Gravity 28, 175011 (2011).
[15] C. Guedes, D. Oriti, and M. Raasakka, Quantization maps, algebra representation and commutative Fourier transform for Lie groups, J. Math. Phys. 54, 083508 (2013).

[16] M. Duflo, Opérateurs différentiels bi-invariants sur un groupe de Lie, Ann. Scient. Éc. Norm. Sup. 10, 265 (1977).

[17] A. Alekseev, A. P. Polychronakos, and M. Smedback, On area and entropy of a black hole, Phys. Lett. B 574, 296 (2003).

[18] L. Freidel and S. Majid, Noncommutative harmonic analysis, sampling theory and the Duflo map in $2+1$ quantum gravity, Classical Quantum Gravity 25, 045006 (2008).

[19] H. Sahlmann and T. Thiemann, Chern-Simons theory, Stokes' theorem, and the Duflo map, J. Geom. Phys. 61, 1104 (2011).

[20] H. Sahlmann and T. Thiemann, Chern-Simons Expectation Values and Quantum Horizons from LQG and the Duflo Map, Phys. Rev. Lett. 108, 111303 (2012).

[21] K. Noui, A. Perez, and D. Pranzetti, Canonical quantization of commutative holonomies in $2+1$ loop quantum gravity, J. High Energy Phys. 10 (2011) 036.

[22] L. Rosa and P. Vitale, On the $\star$-product quantization and the Duflo map in three dimensions, Mod. Phys. Lett. A 27, 1250207 (2012).

[23] G. Vilasi, Hamiltonian Dynamics (World Scientific, Singapore, 2001), p. 440.

[24] D. Alekseevsky, J. Grabowski, G. Marmo, and P. W. Michor, Poisson structures on the cotangent bundle of a Lie group or a principle bundle and their reductions, J. Math. Phys. 35, 4909 (1994).

[25] K. H. Hofmann and A. Mukherjea, On the density of the image of the exponential function, Math. Ann. 234, 263 (1978).

[26] D.Z. Doković and K. H. Hofmann, The surjectivity question for the exponential function of real Lie groups: A status report, J. Lie Theory 7, 171 (1997).

[27] M. Kontsevich, Deformation quantization of Poisson manifolds. 1, Lett. Math. Phys. 66, 157 (2003).

[28] D. Calaque and C. A. Rossi, Lectures on Duflo Isomorphisms in Lie Algebra and Complex Geometry, EMS Series of Lectures in Mathematics (European Mathematical Society, Zurich, Switzerland, 2011).

[29] W. Rühl, The Lorentz Group and Harmonic Analysis (W. A. Benjamin, New York, 1970).

[30] B. C. Hall, Lie Groups, Lie Algebras, and Representations (Springer International Publishing, Switzerland, 2015).

[31] W. Rossmann, Lie Groups, an Introduction Through Linear Groups (Oxford University Press, New York, 2002).

[32] K. H. Hofmann, A memo on the exponential function and regular points, Arch. Math. 59, 24 (1992).

[33] V. G. Kupriyanov and P. Vitale, Noncommutative $\mathbb{R}^{d}$ via closed star product, J. High Energy Phys. 08 (2015) 024.

[34] A. Alekseev and E. Meinrenken, The commutative Weil algebra, Inventiones Mathematicae 139, 135 (2000).

[35] M. Duflo and E. Petracci, Symmetric pairs and Gorelik elements, J. Algebra 313, 125 (2007).

[36] M. Born, A suggestion for unifying quantum theory and relativity, Proc. R. Soc. A 165, 291 (1938).

[37] S. Majid, Foundations of Quantum Groups (Cambridge University Press, Cambridge, England, 1995). 
[38] H. S. Snyder, Quantized space-time, Phys. Rev. 71, 38 (1947).

[39] F. Girelli and E. R. Livine, Scalar field theory in Snyder space-time: Alternatives, J. High Energy Phys. 03 (2011) 132.

[40] J. Lukierski and H. Ruegg, Quantum kappa Poincare in any dimension, Phys. Lett. B 329, 189 (1994).

[41] S. Zakrzewski, Quantum Poincare group related to the kappa -Poincare algebra, J. Phys. A 27, 2075 (1994).

[42] S. Majid and H. Ruegg, Bicrossproduct structure of kappa Poincare group and noncommutative geometry, Phys. Lett. B 334, 348 (1994).

[43] J. Kowalski-Glikman, De sitter space as an arena for doubly special relativity, Phys. Lett. B 547, 291 (2002).

[44] A. Agostini, G. Amelino-Camelia, and F. D'Andrea, Hopf algebra description of noncommutative space-time symmetries, Int. J. Mod. Phys. A 19, 5187 (2004).

[45] F. Girelli, E. R. Livine, and D. Oriti, 4d deformed special relativity from group field theories, Phys. Rev. D 81, 024015 (2010).

[46] A. Perez and C. Rovelli, Spin foam model for Lorentzian general relativity, Phys. Rev. D 63, 041501 (2001).

[47] A. O. Barut and R. Raczka, Theory of Group Representations and Applications (Polish Scientific Publishers, Waszawa, 1980).
[48] M. Arzano, D. Latini and M. Lotito, Group momentum space and hopf algebra symmetries of point particles coupled to $2+1$ gravity, SIGMA 10, 079 (2014).

[49] D. Oriti and M. Raasakka, Quantum mechanics on SO(3) via non-commutative dual variables, Phys. Rev. D 84, 025003 (2011).

[50] M. Bohm and G. Junker, Path integration over compact and noncompact rotation groups, J. Math. Phys. 28, 1978 (1987).

[51] A. Inomata and G. Junker, Path integrals and lie groups, in Noncompact Lie Groups and Some of their Applications, NATO ASI Series (Kluwer Academic Publishers, Dordrecht, 1994), p. 199.

[52] N. J. Vilenkin and A. U. Klimyk, Representation of Lie Groups and Special Functions (Kluwer Academic Publishers, Dordrecht, 1993).

[53] J.S. Dowker, Quantum mechanics on group space and Huygens' principle, Ann. Phys. (N.Y.) 62, 361 (1971).

[54] M. Finocchiaro and D. Oriti, Spin foam models and the Duflo map, arXiv:1812.03550.

[55] E. Livine and D. Oriti, Implementing causality in the spin foam quantum geometry, Nucl. Phys. B663, 231 (2003). 